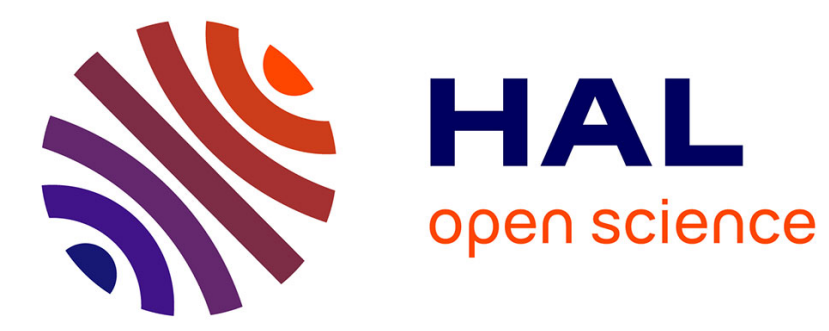

\title{
On accounting for speckle extinction via DIC and PCA
}

R Vargas, R B Canto, François Hild, Stéphane Roux

\section{To cite this version:}

R Vargas, R B Canto, François Hild, Stéphane Roux. On accounting for speckle extinction via DIC and PCA. Optics and Lasers in Engineering, 2022, 149, pp.106813. 10.1016/j.optlaseng.2021.106813 . hal-03358220

\section{HAL Id: hal-03358220 \\ https://hal.science/hal-03358220}

Submitted on 29 Sep 2021

HAL is a multi-disciplinary open access archive for the deposit and dissemination of scientific research documents, whether they are published or not. The documents may come from teaching and research institutions in France or abroad, or from public or private research centers.
L'archive ouverte pluridisciplinaire HAL, est destinée au dépôt et à la diffusion de documents scientifiques de niveau recherche, publiés ou non, émanant des établissements d'enseignement et de recherche français ou étrangers, des laboratoires publics ou privés. 


\title{
On accounting for speckle extinction via DIC and PCA
}

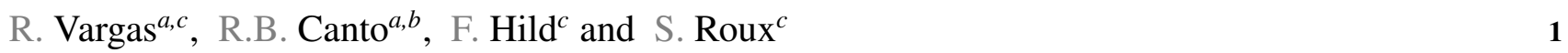

${ }^{a}$ Federal University of São Carlos (UFSCar), Graduate Program in Materials Science and Engineering (PPGCEM), 13565-905, São Carlos-SP, 2

Brazil

${ }^{b}$ Federal University of São Carlos (UFSCar), Department of Materials Engineering (DEMa), 13565-905, São Carlos-SP, Brazil, Brazil

${ }^{c}$ Université Paris-Saclay, ENS Paris-Saclay, CNRS, LMT - Laboratoire de Mécanique et Technologie, 91190 Gif-sur-Yvette, France

\section{ARTICLE INFO}

\section{Keywords:}

Brightness conservation

Digital Image Correlation (DIC)

High temperature

Principal Component Analysis (PCA)

Wedge Splitting Test (WST)

\begin{abstract}
A B S T R ACT
Digital Image Correlation (DIC) is a full-field measurement technique that generally relies on brightness conservation principles. This work aims to analyze cases in which the speckle pattern vanished after heating, thereby severely altering the brightness. Sets of images acquired during 6 experiments were registered and then used to obtain optimal sets of reference images for each time step via Principal Component Analysis (PCA). The proposed methodology significantly reduced the gray level residuals and provided insight into brightness variations that occurred in the analyzed cases. It was possible to study cases with severe speckle pattern changes by updating the reference image when standard DIC did not converge and using the PCA methodology to obtain optimal reference states.
\end{abstract} 8

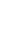

(

(1)

9

\footnotetext{
*Corresponding author

@email@institution (R. Vargas)

ORCID(s): 0000-0002-1711-9804 (R. Vargas); 0000-0002-9286-9912 (R.B. Canto); 0000-0001-5553-0066 (F. Hild); 


\section{Introduction}

Digital Image Correlation (DIC) is a measurement technique that allows displacement fields to be measured [1, 2]. Similar (if not identical) frameworks can be used for different types of images and under several magnifications [3] and modalities [4]. Moreover, one additional advantage is its applicability under severe conditions since contact is not needed [5]. For standard conditions, the gray level of each pixel is directly related to the brightness of the imaged domain of the sample. The most common assumption is the conservation of brightness [6] (i.e., the same gray level may be found in successive images but at different locations). However, such hypothesis is not always satisfied (e.g., lighting variations or changes in specimen color may occur).

Another interesting and complementary tool is the Principal Component Analysis (PCA), one technique that identifies orthogonal modes for data reduction and is commonly used to extract dominant patterns from high-dimensional data [7]. Due to its generality, PCA is used in many applications. Concerning DIC, if small displacements occur, it can be applied directly on images to extract kinematic bases for DIC analyses [8]. It can also be used for filtering out noise from measured displacement fields $[9,10,11]$. Other applications include estimating dynamic properties in viscoelastic materials [12], evaluating vibrations modes of cantilever plate [13], and clustering displacement vectors to analyze fracture mechanisms in rocks [14]. Berny et. al [15] showed how proper generalized decomposition (PGD) could be used to construct on the fly modal bases within a DIC framework.

Usually, DIC is based upon the brightness conservation hypothesis in images in which the digital levels are related to the brightness in the visible spectrum $[6,1]$. However, the same framework can be extended to other types of images in which the gray levels may encode a physical information [4]. Atomic force microscopy images, in which digital levels are related to heights, were used to estimate stress intensity factors [16]. Similarly, confocal microscopy images led to measurements of in and out-of-plane displacements for membranes [17]. Infrared images can also be registered to measure displacement fields and Lagrangian temperature fields $[18,19]$.

Several studies dealt with brightness corrections, since in practice it is not always feasible to maintain the same conditions for every acquired image throughout experiments. Peng et al. [20] assumed linear changes, Gu et al. [21] used a top-hat correction for tackling uneven intensity changes, and Hu et al. [22] proposed a position-based intensity change model. The so-called zero-mean normalization of images is another common choice [1, 23, 24, 25]. Other corrections are found when the brightness of some pixels saturated [26, 27]. Sciuti et al. [28] analyzed the effects of brightness and contrast corrections using one Q8 element or the same mesh applied for the kinematics for studying $\mathrm{MgO}$ curing inside a climatic chamber. Archer et al. [29] accounted for brightness inversion of the pattern at very high temperatures. Hallo et al. [30] used low order polynomials for brightness and contrast corrections to detect and quantify damage in optics of a high energy laser facility.

Although studies on the pattern effect can be found [31], to the authors' best knowledge, no work explicitly dealing 
with its extinction during tests exists. One example of severe changes in images from an Italian glacier and from Chad's desert was reported and analyzed under different DIC frameworks for comparison purposes [32]. In the following, the case of severe extinction of speckle patterns is addressed using PCA to find reduced spatiotemporal bases of reference states. The methodology is first introduced in Section 2 and then applied to measure Notch Opening Displacements (NODs) in Wedge Splitting Tests (WSTs) performed at room temperature and $600^{\circ} \mathrm{C}$ (Section 3). In these tests, the speckle pattern changed in addition to uneven variations of brightness due to shadows created during loading. The final case of Section 4 deals with a dilatometry experiment in which severe speckle extinction occurred.

\subsection{Motivation}

The experiment reported in this section is a dilatometry test in a furnace during which the sole loading consisted of a heating ramp $\left(\approx 2^{\circ} \mathrm{C} / \mathrm{min}\right.$ ), dwell time (for $1.5 \mathrm{~h}$ at $650^{\circ} \mathrm{C}$ ) and a cooling down ramp (Figure 1) applied to an alumina-rich castable with mullite-zirconia aggregates [33]. In this paper, the same nomenclature as in Ref. [33] is used; the first test being on sample MZ1-S1450G. "MZ" refers to mullite-zirconia aggregates followed by the test number. The number after "S" gives the sintering temperature expressed in ${ }^{\circ} \mathrm{C}$. Last, "G" indicates the presence of lateral grooves to guide the crack in wedge splitting tests (WSTs).

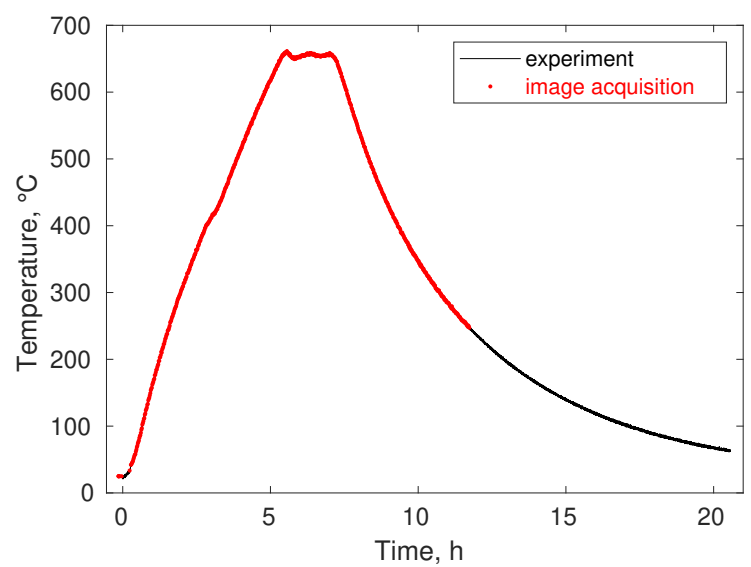

Figure 1: Temperature measured with a thermocouple located in the middle of sample MZ1-S1450G (Figure 2) for the dilatometry test and the 690 time steps when images were acquired

A total of 690 images was acquired during the major part of the test. The hardware parameters are summarized

in Table 1. Since the dilatometry test was performed on sample MZ1-S1450G that was initially fractured at room 68 temperature, very similar parameters were kept. The main difference was the acquisition rate, since the duration for the dilatometry experiment was considerably longer and the observed phenomena were not expected to change in smaller time increments. Moreover, at the beginning of each experiment, characteristic pattern sizes were measured 
as the width at mid-height of the auto-correlation function.

\section{Table 1}

DIC hardware parameters

\begin{tabular}{|l|l|}
\hline \hline Camera & Canon 70D \\
Gefinition & $5472 \times 3648 \mathrm{px}$ \\
Lens & 8 bits \\
Field of view & Canon Macro 100 \\
Image scale & $104 \times 69 \mathrm{~mm}^{2}$ \\
Stand-off distance & $50 \mathrm{~cm}$ \\
Image acquisition rate & dilatometry: 0.017 fps | WSTs: 0.1 fps \\
Patterning technique & sprayed paints \\
Pattern size & 5 px (MZ1-S1450G, MZ2-S1400, MZ3-S1400G) \\
& 12 px (MZ4-S1450, MZ5-S1450G) \\
\hline \hline
\end{tabular}

Specimen MZ1-S1450G (Figure 2(a)) was a post-mortem WST sample that was previously tested at room temperature [33] (turned $90^{\circ}$ counterclockwise). The highlighted region of interest (ROI) in Figure 2(b) shows that the speckle pattern had good contrast at the beginning, but that it mostly vanished during the dilatometry test (Figures 2(c,d)) pos75 sibly due to thermal degradation of the paint and diffusion in the sample. These ROIs will be used as motivation of the methodology proposed herein to measure displacement fields via DIC and extract the coefficient of thermal expansion 


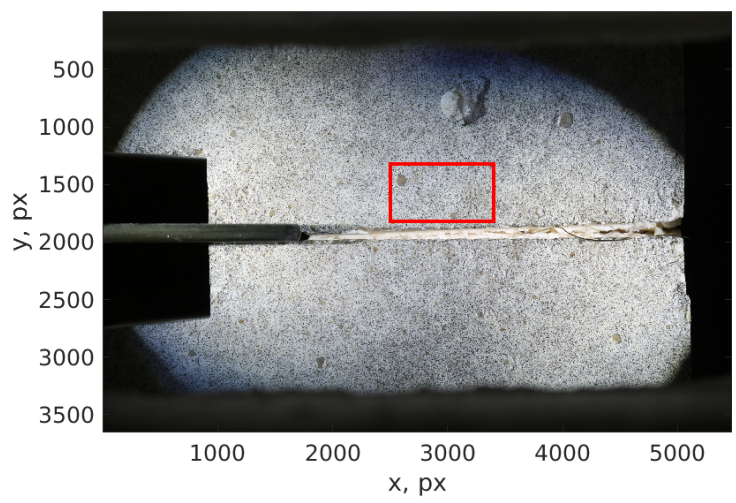

(a)

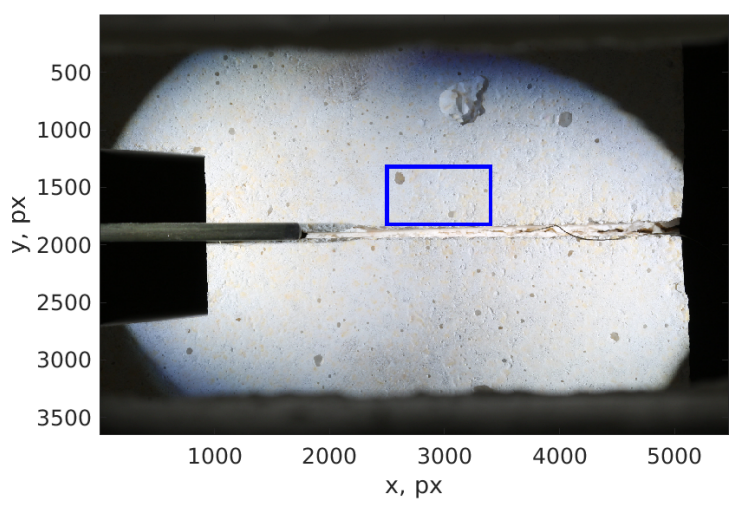

(c)

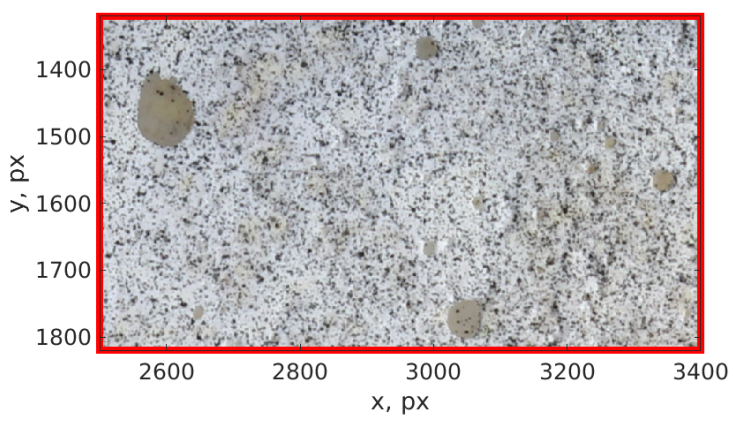

(b)

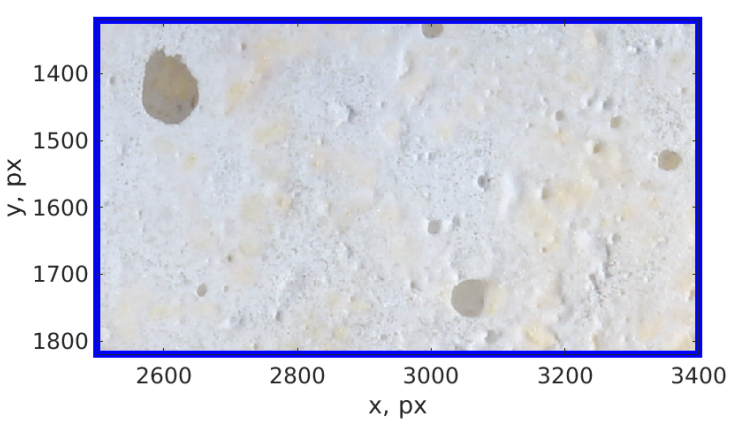

(d)

Figure 2: (a) Reference image prior to heating sample MZ1-S1450G (after the WST was performed). The ROI (red rectangle) is shown in sub-figure (b). (c) Last image after cooling down. The corresponding ROI (blue rectangle) is shown in sub-figure (d). Note that the speckle pattern almost disappeared during heating

To quantify the offset to gray level conservation, the histograms of the 8-bit encoded ROIs shown in Figures 2(b,d) 


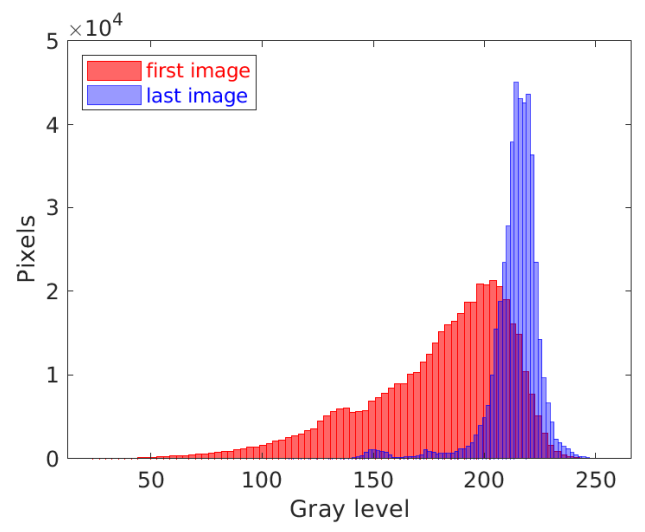

Figure 3: Gray level histograms of the 8-bit ROls highlighted in Figures 2(b,d)

For the proposed methodology, a first kinematic correction is needed. It was chosen to perform one DIC step with a very coarse mesh (Figure 4(a)) for the 690 pictures. Rigid body translations and first order displacement gradients were used as a reduced kinematic basis (i.e., six degrees of freedom). Two DIC analyses were performed. First, incremental registrations (i.e., the deformed configuration of the $n$-th registration becomes the reference configuration of the $(n+1)$-th registration) were carried out. Its aim was to check whether DIC could be run under such challenging conditions. Second, direct registrations (the reference image is the first of the series) were run when initialized by the previous analysis. In the present case, such approach could not converge for the whole set of images and thus the socalled quasi-direct approach was introduced. Every time divergence was detected (i.e., five consecutive iterations with increasing gray level residuals), the reference image for DIC calculations was updated with its estimate for the previous time step (i.e., the deformed image corrected by the measured displacement field). For the six analyzed experiments, the first incremental step took about $0.45 \mathrm{~s}$ per analyzed image, which reduced to $0.36 \mathrm{~s}$ for quasi-direct DIC with the improved initialization. 


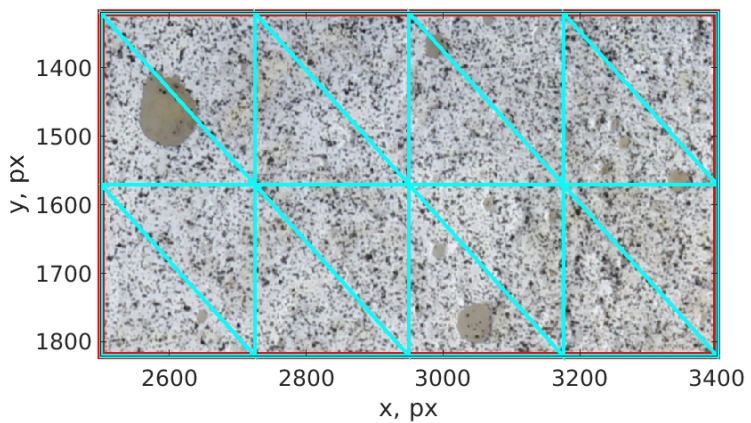

(a)

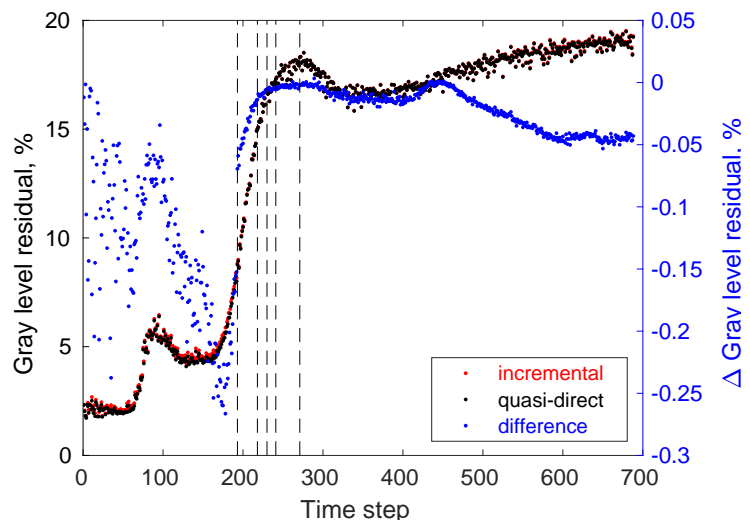

(b)

Figure 4: (a) Coarse mesh for the first kinematic correction. (b) Normalized RMS gray level residual for the preliminary DIC step with incremental and quasi-direct approaches. The vertical dashed lines depict updates of reference images for quasi-direct DIC

The normalized gray level residuals from this first analysis (Figure 4(b)), always calculated with respect to the very first reference state, reached a level of $2 \%$ of the dynamic range of the reference picture at the beginning of the test, which is regarded as good for common DIC analyses [4]. However, as the test proceeded, the residuals considerably increased up to $12 \%$ since gray level conservation is no longer satisfied (Figure 3). As indicated by the dashed vertical lines in Figure 4(b), five updates were needed, mostly concentrated between images 200 and 300 when the speckle vanished. The difference between incremental and quasi-direct approaches was less than $0.5 \%$ for every time step. Given the fact that measurement uncertainties are cumulated in incremental approaches, this difference was deemed small since gray levels significantly varied (Figure 2(b,d)). Conversely, it is believed that incremental DIC is more secure when severe gray level variations occur. In the present case, a very coarse mesh was selected to capture mean dilatational strains so that the quasi-direct method could converge as well. The quasi-direct DIC results were selected as reference for the following analyzes of Section 2 .

\subsection{Gray level conservation}

DIC analyses are commonly based on the hypothesis that, in the absence of noise, the same gray level $f\left(\mathbf{x}, t_{0}\right)$ of pixel $\mathbf{x}$ in the reference state (at time $t_{0}$ ) is to be found in a deformed state $g$ (at time $t$ ) but at a different position

$$
f\left(\mathbf{x}, t_{0}\right)=g(\mathbf{x}+\mathbf{u}(\mathbf{x}, t), t)
$$

where $\mathbf{u}$ is the displacement vector. In this framework, a sequence of images is registered with the same reference to progressively measure displacement fields by minimizing the global residual $\varrho^{2}$ at each acquisition time (i.e., via 
instantaneous analyses)

$$
\rho^{2}(t)=\sum_{\mathbf{x} \in \mathrm{ROI}} \rho(\mathbf{x}, t)^{2} \quad \text { with } \quad \rho(\mathbf{x}, t)=f\left(\mathbf{x}, t_{0}\right)-g(\mathbf{x}+\mathbf{u}(\mathbf{x}, t), t)
$$

evaluated over the whole region of interest (ROI) if global approaches are followed [34, 35]. From such analyses, the $\mathbf{1 0 8}$ overall quality of DIC is measured with the root mean square (RMS) residual $\varrho$ normalized by the dynamic range of $\mathbf{1 0 9}$ $f\left(\bullet, t_{0}\right)$.

Since the hypothesis of gray level conservation was not satisfied in the present case (Figure 3), it is proposed to modify Equation (2) with a time dependent reference state that has to be updated, denoted as $f(\mathbf{x}, t)$, so that the pixel-wise residual reads

$$
\rho(\mathbf{x}, t)=f(\mathbf{x}, t)-g(\mathbf{x}+\mathbf{u}(\mathbf{x}), t)
$$

The proposed modification accounts for the actual surface texture at any instant $t$, even if it is changing over time. The difficulty however is that $f(\mathbf{x}, t)$ is not known a priori and is to be determined along with the displacement field $\mathbf{u}(\mathbf{x})$. The next section is devoted to the description of the way to transition from $f\left(\mathbf{x}, t_{0}\right)$ to $f(\mathbf{x}, t)$. The analyses discussed herein were performed within the Correli 3.0 framework [36] in which a finite-element approach was implemented (Table 2). The measured displacement fields were discretized with 3-noded (T3) finite elements (Figure 4(a)). Coarse meshes were used since finer details were not needed for thermal expansion and notch opening displacement measurements. For each presented case, 100 images were available prior to the experiment for uncertainty quantification. The displacement noise-floor $\sigma_{u}$ will be given in the text for each analyzed case.

\section{Table 2}

DIC analysis parameters

\begin{tabular}{|l|l|}
\hline \hline DIC software & Correli $3.0[36]$ \\
Image filtering & none \\
Element length & dilatometry: $170 \mathrm{px} \mid$ NOD: $140 \mathrm{px}$ \\
Shape functions & linear (T3) \\
Meshes & see Figures 4 and 5 \\
Matching criterion & sum of squared differences \\
Interpolant & cubic \\
Displacement noise-floor & see text $\left(\sigma_{u}\right)$ \\
\hline \hline
\end{tabular}

\subsection{Accounting for pattern extinction}

Instead of choosing a single reference image, an alternative route has to be followed in the present case since gray level conservation was not satisfied. It is worth noting that at the end of any registration under the assumption of gray 
level conservation, the image of the deformed configuration corrected by the measured displacement field, namely $\widetilde{g}(\mathbf{x}, t)=g(\mathbf{x}+\mathbf{u}(\mathbf{x}, t), t)$, is a very good approximation of $f\left(\mathbf{x}, t_{0}\right)$. Consequently, in a set of $n_{t o t}$ images in deformed states during the experiment, one ends up having $n_{\text {tot }}+1$ estimates of the reference state. Such observation has led to the introduction of a denoised reference image in spatiotemporal DIC [37].

The unknown $f(\mathbf{x}, t)$ images have to be evaluated to account for pattern extinction. The first step of such analysis consists in running DIC analyses by assuming gray level conservation. At the end of such step, if convergence was observed, the series of corrected images $\widetilde{g}(\mathbf{x}, t)$ provide first order estimates of $f(\mathbf{x}, t)$. Consequently, a first movie is available. It corresponds to the temporal stacking comprising $\left.f\left(\mathbf{x}, t_{0}\right) \approx \widetilde{g}\left(\mathbf{x}, t_{0}\right)\right)$ and all $n=1, \ldots, n_{\text {tot }}$ corrected images $\widetilde{g}\left(\mathbf{x}, t_{n}\right)$. A Singular Value Decomposition (SVD) is performed on such movie $\widetilde{g}$ with a space-time separation such that

$$
\tilde{g}(\mathbf{x}, t)=\sum_{i=1}^{n_{t o t}+1} \zeta_{i}^{1}(\mathbf{x}) \lambda_{i}^{1} \tau_{i}^{1}(t)
$$

where $\zeta_{i}^{1}$ denotes the $i$-th normalized spatial mode, $\tau_{i}^{1}$ the corresponding temporal mode, and $\lambda_{i}^{1}$ the singular value. Care should be taken in this step since the memory usage for such decomposition is directly proportional to the image size and number of time steps. A reduced representation of $\widetilde{g}$ using PCA is denoted by $\varphi^{1}$; it is obtained as a truncation of the SVD of $\widetilde{g}$

$$
\varphi^{1}(\mathbf{x}, t)=\sum_{i=1}^{n_{t r}} \zeta_{i}^{1}(\mathbf{x}) \lambda_{i}^{1} \tau_{i}^{1}(t)
$$

with the truncation number $n_{t r}$ being less than $n_{t o t}+1$

since the number of pixels in the ROI is assumed to be greater than the number of images. The spatial and temporal modes correspond to the left-singular and right-singular vectors of the singular value decomposition of $\widetilde{g}$.

The truncation step of $\widetilde{g}$ after having a first evaluation of the displacement field $\mathbf{u}^{0}(\mathbf{x}, t)$ is shown in Algorithm 1. It requires the standard displacement uncertainty $\sigma_{u}$ to be quantified for the analyzed test. In this approach, the truncation number $n_{t r}$ is set so that the last added mode leads to a displacement increment whose instantaneous fluctuation $\Delta_{u}(t)$ with respect to the previous estimate is less than the standard displacement uncertainty for every time step. It is worth noting that $\Delta_{u}(t)$ is initialized from the first estimate of the displacement field since if its instantaneous RMS is less than $\sigma_{u}$ this approach would be meaningless. Although the displacement uncertainty is likely to change throughout the test with degrading speckle patterns, $\sigma_{u}$ was kept constant for the sake of simplicity. Moreover, since a very good initialization is used, each analysis takes about $0.25 \mathrm{~s}$. Hereafter, all time steps were analyzed to illustrate the displacement residuals but much time could be gained if the analysis proceeded to the next truncation at the first difference becoming greater than the uncertainty. 


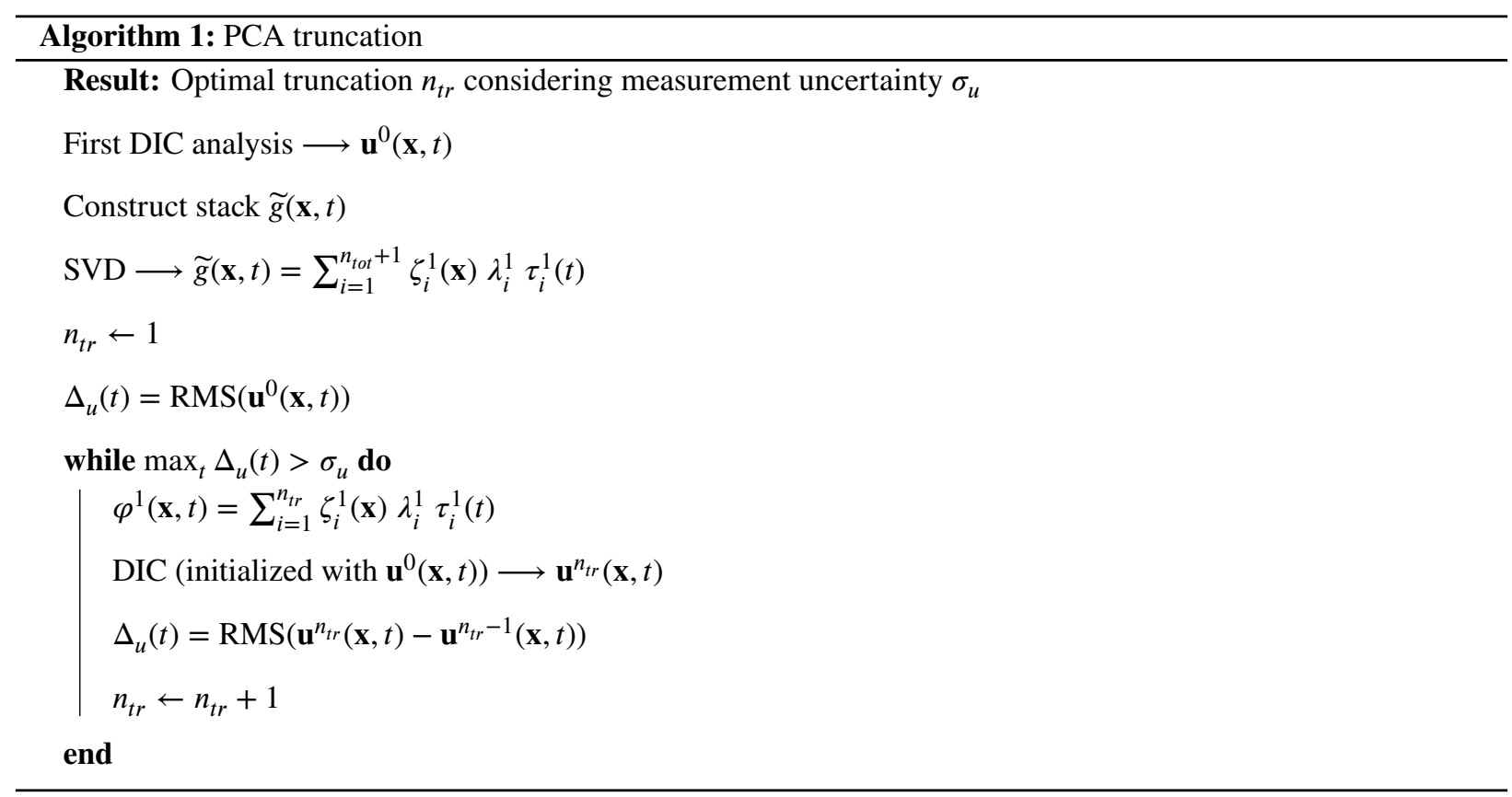

Once the truncation was performed, the estimates of $f\left(\mathbf{x}, t_{n}\right)$ are obtained by using Equation (5). A new DIC anal- 141 ysis is run using the new references to update the estimate of the displacement fields and corresponding gray level 142 residuals. To speed up this new step, it is initialized with the previously measured displacements. This strategy results $\mathbf{1 4 3}$ in an analysis as fast as Algorithm 1, taking $0.25 \mathrm{~s}$ per analyzed image. At the end of this step, a new stack $\tilde{g}^{1}$ is available and the $n_{t o t}+1$ modes can be re-evaluated and truncated with the same number $n_{t r}$. These steps are per- 145 formed until the RMS difference between displacement fields obtained from two successive steps becomes less than 146 the standard displacement uncertainty (Algorithm 2). One advantage of the present algorithms is the possibility to $\mathbf{1 4 7}$ easily implement them non-intrusively in any DIC code (i.e., one only needs to change the set of images given as in- 148 put). More robust implementations could be thought to further explore the orthogonality of the modes given by SVD, 149 for instance, using the gradients of the spatial modes directly in the DIC Hessian. However, such approaches would 150 require more advanced knowledge from the user to change some routines inside the DIC code. 


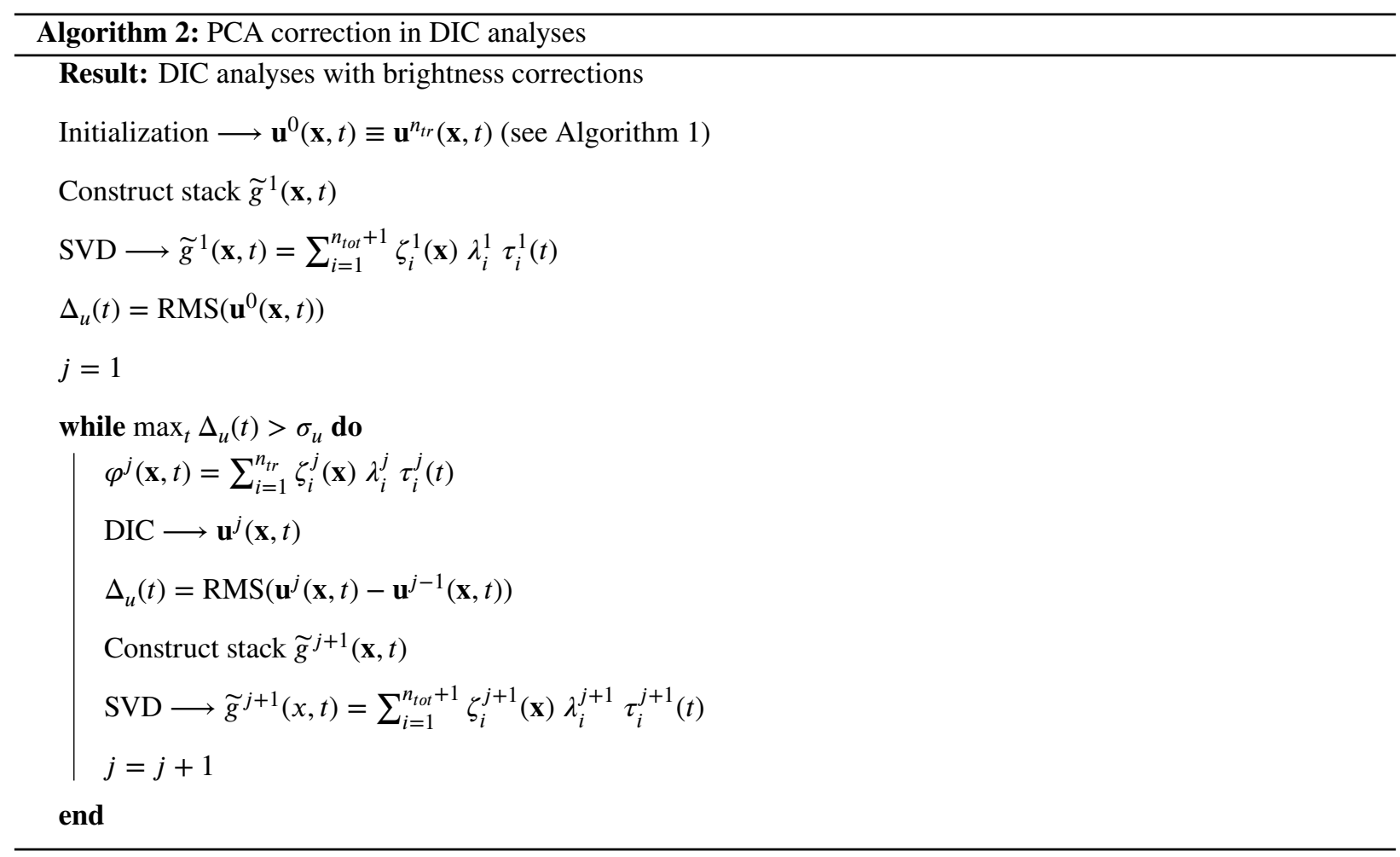

\section{Notch opening displacement calculations}

In this section, the proposed brightness correction is tested on 5 Wedge Splitting Tests (WSTs) in which the first 155 interest was measuring the Notch Opening Displacement (NOD) to evaluate the work of fracture [33]. The NOD 156 corresponds to the difference of mean displacement of the two $400 \times 400$ px ROIs shown in Figure 5(a) (yellow boxes). 157 The reduced kinematic basis for each ROI consisted of six degrees of freedom (i.e., mean translation and first order 158 gradients for each displacement component). This choice was made to have minimal couplings between the sought kinematics and the reference image correction. First, a room temperature test (MZ1-S1450G), in which the previous corrections were not expected to be needed, is analyzed. Then, four other experiments performed at $600^{\circ} \mathrm{C}$ are studied, 161 in which direct DIC could not converge in the last two. For these very challenging cases, an intermediate route was followed, namely, quasi-direct DIC that will be compared to incremental DIC, which could always converge in any of 163 the reported cases even with severe pattern extinction. The rate at which the pattern evolved was observed to be slow as compared to the acquisition rate and hence the incremental analyses could converge. However, this requires a large 165 number of images and thus the cumulative displacements come unavoidably together with an accumulation of errors that degrade the overall uncertainty. 


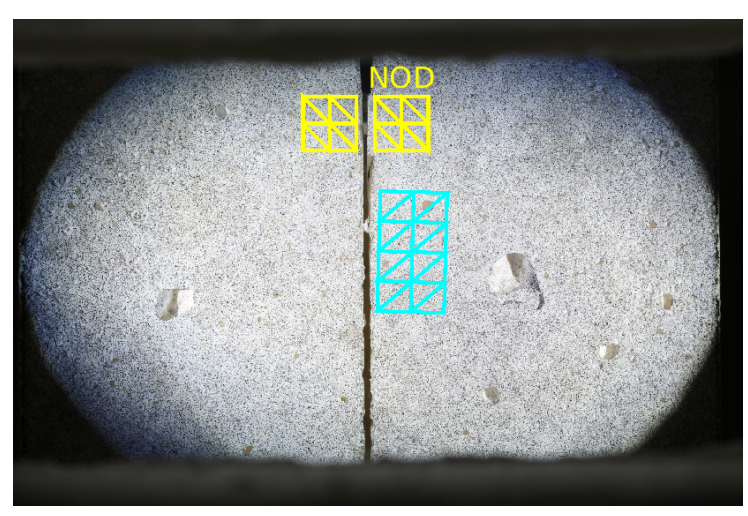

(a)

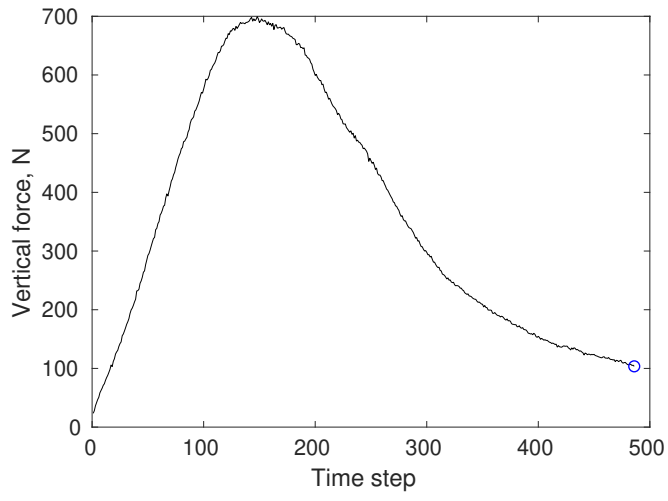

(b)

Figure 5: (a) Reference image for the room temperature WST (MZ1-S1450G). The yellow meshes depict the ROIs for NOD calculations. The sample is the same as that tested post-mortem in the dilatometry experiment (Figure 2), and the corresponding mesh is shown in cyan. (b) Applied force vs. time steps of image acquisitions. The blue circle depicts the final unloading level used for the evaluation of the work of fracture (and the end of the present analyses)

The loading curve for the room temperature WST is shown in Figure 5(b) for illustration purposes. The work of 168 fracture was obtained from the NOD vs. splitting force curve. The splitting force was assessed from the applied force $\mathbf{1 6 9}$ (i.e., multiplied by $5.715[38,39]$ ). The work-conjugate quantity is the so-called crack mouth opening displacement 170 (CMOD). In the present case, it could not be measured directly since the top part of the sample was not visible (Fig- 171 ure 5(a)). However, the NOD could be measured and a linear relationship between NOD and CMOD was shown to 172 apply in the propagation regime (i.e., $\mathrm{CMOD}=1.62 \mathrm{NOD}$ ). The fracture energy $\Gamma_{c}^{N O D}$ was then calculated by divid- 173 ing the work of fracture by twice the projected cracked area, considering a propagation throughout the vertical middle $\mathbf{1 7 4}$ plane [33].

To illustrate the challenges involved hereafter, Figure 6 shows the first and last images in each $\tilde{g}$ stack after direct 176 (or quasi-direct) analyses. The same color map was used in which the gray levels ranged from 0 and 255. Some pixels 177 of the NOD ROIs were masked because their gray levels saturated. The changes in brightness are observed in all high $\mathbf{1 7 8}$ temperature experiments (Figure 6(d,f,h,j)) and a vertical brightness gradient is visible in the last image with a darker 179 top region. Moreover, the pattern size for the last two cases was coarser than in the three first experiments [33]. 


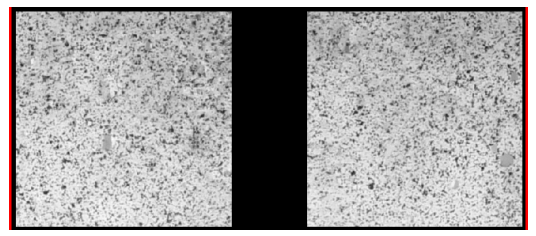

(a)

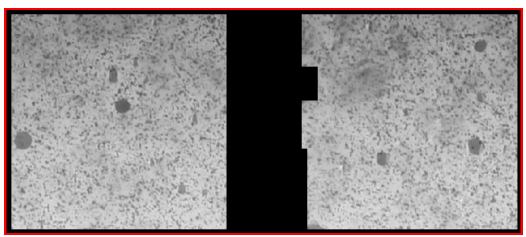

(c)

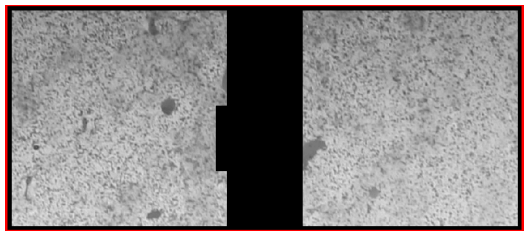

(e)

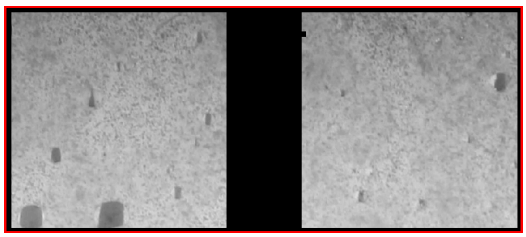

(g)

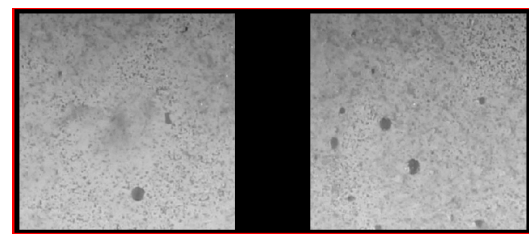

(i)

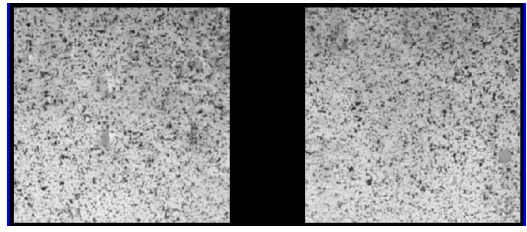

(b)

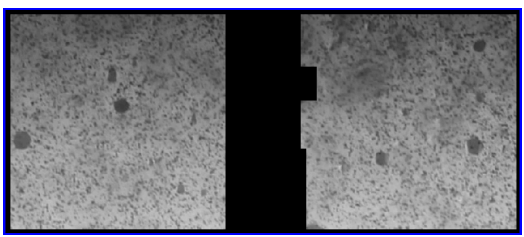

(d)

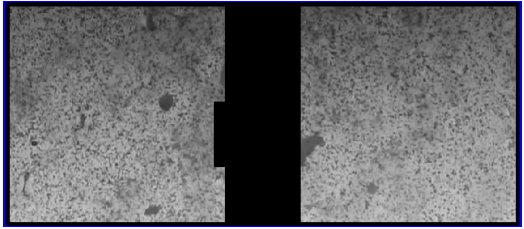

(f)

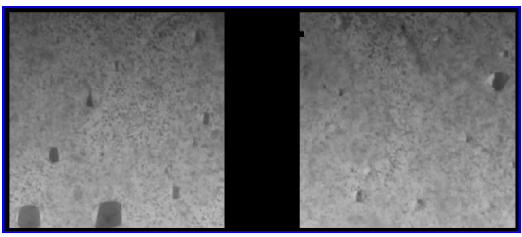

(h)

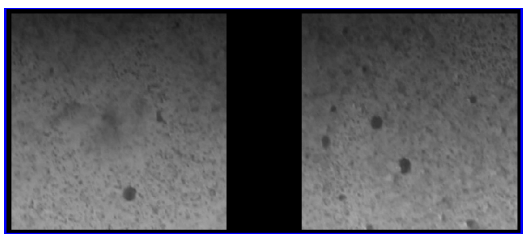

(j)

Figure 6: First (left column) and last (right column) images (ROls for NOD calculations) in the $\widetilde{g}$ stacks for the room temperature experiment: MZ1-S1450G (a,b), and for the $600^{\circ} \mathrm{C}$ experiments: MZ2-S1400 (c,d), MZ3-S1400G (e,f), MZ4-S1450 (g,h), and MZ5-S1450G (i,j)

The maximum and minimum gray levels shown in Figure 6 are reported in Table 3, along with the test temperature 
each analyzed time step.

\section{Table 3}

Minimum and maximum gray levels for the first and last images of the 8-bit stacks $\widetilde{g}$, the temperature in which the experiment was performed, and the time between the acquisition of both images

\begin{tabular}{|c|c|c|c|c|}
\hline Experiment & Test temperature & First image & Last image & Time between first and last images \\
\hline \hline MZ1-S1450G & room temperature & $27 \mid 255$ & $23 \mid 253$ & 45 min \\
\hline MZ2-S1400 & $600^{\circ} \mathrm{C}$ & $67 \mid 235$ & $40 \mid 205$ & 65 min \\
\hline MZ3-S1400G & $600^{\circ} \mathrm{C}$ & $71 \mid 235$ & $37 \mid 215$ & $110 \mathrm{~min}$ \\
\hline MZ4-S1450 & $600^{\circ} \mathrm{C}$ & $74 \mid 229$ & $40 \mid 200$ & $115 \mathrm{~min}$ \\
\hline MZ5-S1450G & $600^{\circ} \mathrm{C}$ & $44 \mid 238$ & $24 \mid 183$ & $135 \mathrm{~min}$ \\
\hline
\end{tabular}

\subsection{Room temperature experiment (MZ1-S1450G)}

First, the room temperature test MZ1-S1450G is analyzed. The components obtained via SVD of the first stack of $\mathbf{1 8 7}$ images $\widetilde{g}$ are shown in Figure 7. For this case, no reference update was needed for quasi-direct DIC (i.e., it is equivalent 188 to direct DIC). The first eigenvalue was almost two orders of magnitude higher than the second one (Figure 7(a)) and 189 its temporal component was virtually constant throughout the test (Figure 7(b)). A light background and dark spots of 190 the speckle pattern are visible in the first spatial component (Figure 7(c)). The second mode has a temporal component 191 in phase with the vertical displacement during the test. The corresponding spatial component shows a vertical gradient of gray levels, which is consistent with illumination variations toward the dark region that can be seen on the top of 193 the reference image (Figure 5). 


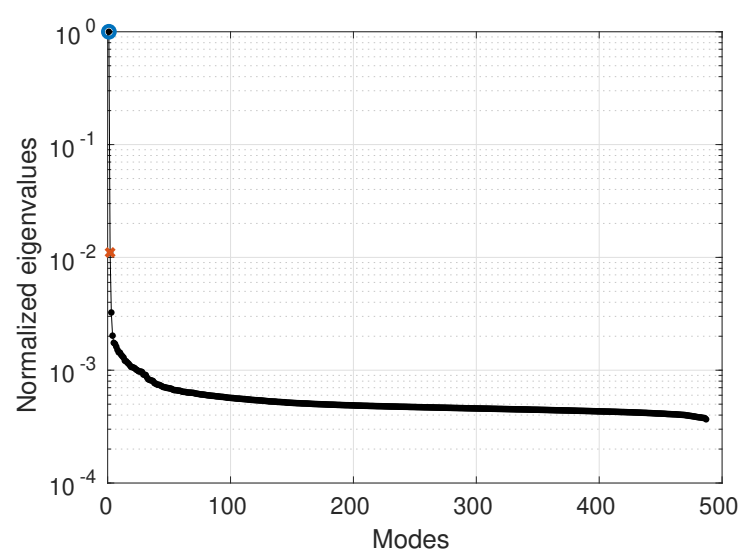

(a)

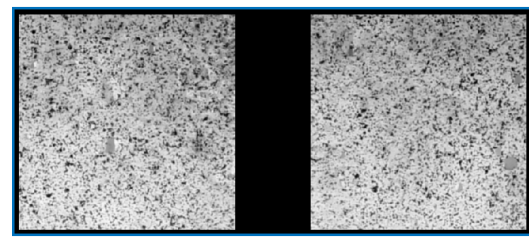

(c)

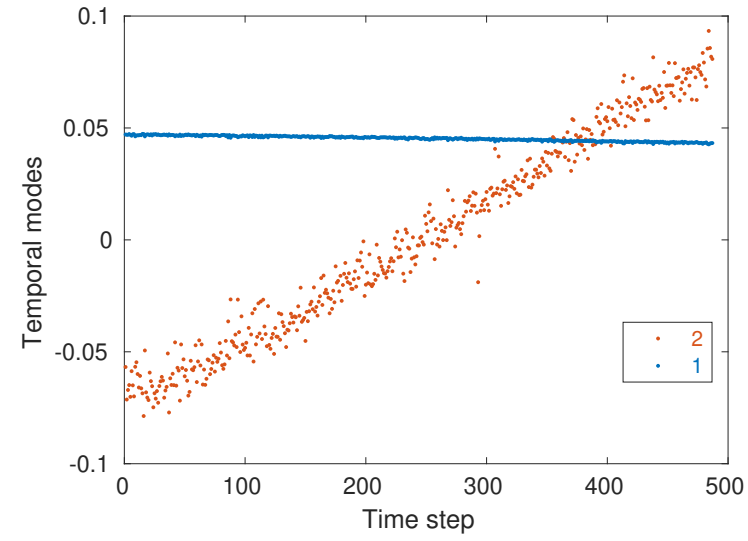

(b)

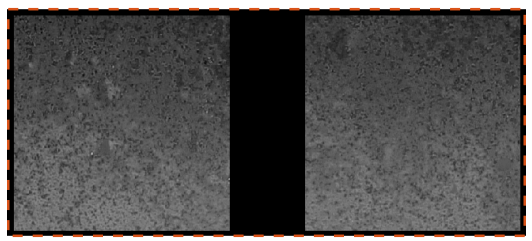

(d)

Figure 7: (a) Normalized eigenvalues of the first SVD of $\widetilde{g}$ for the room temperature WST (MZ1-S1450G). (b) First two temporal modes and (c-e) corresponding spatial modes of the two ROls used for NOD measurements (Figure 5)

For the present and following cases, 100 reference images were acquired prior to each experiment for which the 195 displacement uncertainty was quantified [33]. The standard displacement uncertainty is such that $\sigma_{u}=1.2$ centipixel 196 (i.e., $10^{-2} \mathrm{px}$, denoted as cpx hereafter). When Algorithm 1 was run, it converged in one iteration. Therefore, together 197 with the result of a constant temporal amplitude for the first mode, one single reference image was already well suited. 198 This result was expected since no speckle extinction was observed. The gray level residuals for the truncation and the 199 displacement differences are shown in Figure 8. The proposed correction considerably reduced the gray level residuals, 200 while not altering much the measured displacements. It is worth mentioning that if the second mode was also used, 201 the gray level residuals would become virtually constant around $1 \%$, with no significant gains. The very first iteration was also sufficient to achieve a displacement update less than the uncertainty level. Similar results were observed for Algorithm 2, which also converged in one single iteration (Figure 8(c)). 


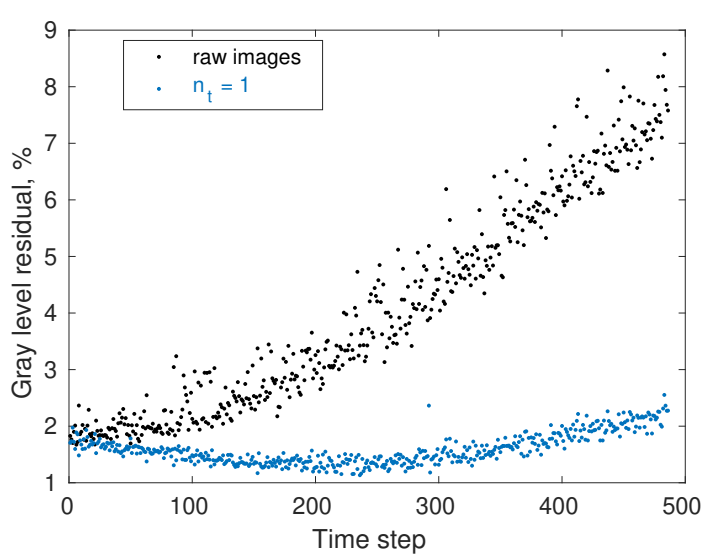

(a)

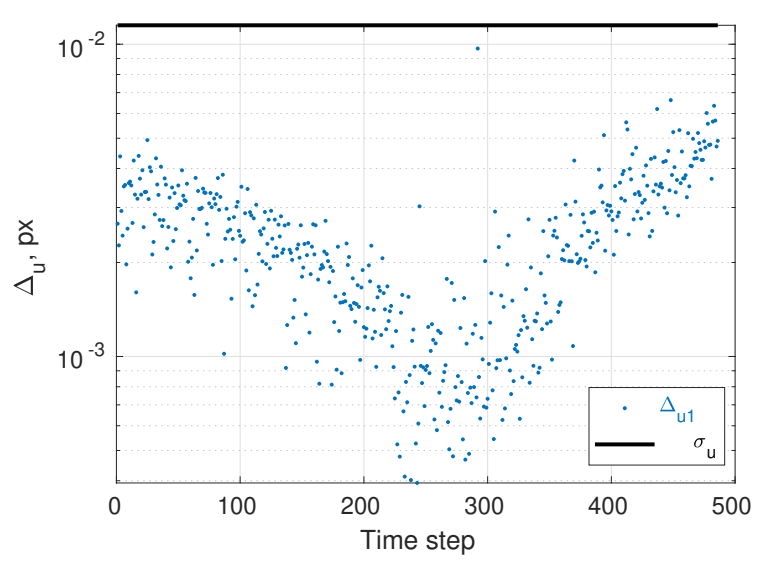

(b)

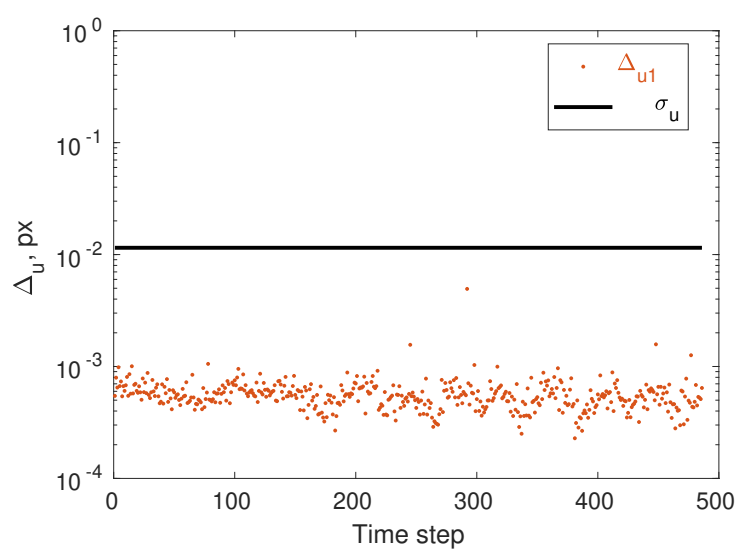

(c)

Figure 8: (a) Normalized RMS residuals for the room temperature WST (MZ1-S1450G) using PCA-based references $\varphi^{1}$. (b) RMS difference between displacements for each tested truncation (Algorithm 1) and (c) for the proposed PCA correction (Algorithm 2). $\sigma_{u}$ is shown in solid black line

The NOD history is displayed in Figure 9. Although the mode I NODs (Figure 9(a)) were used for the fracture 205 energy calculation, the mode II component (in-plane shearing, Figure 9(b)) was also evaluated and used to check for 206 pure mode I assumptions. It was confirmed that the WST was predominantly in mode I. Such conclusion could be 207 drawn for all WSTs analyzed herein. The corresponding results will not be shown for the high temperature tests.

First, incremental DIC was run (in which the reference state was updated for every pair of registered image as explained in Section 2.1). Direct calculations were then performed, initialized with the displacement fields obtained from the incremental approach but now using the first reference image. From incremental to direct DIC, a significant difference is observed between both NODs (with an RMS difference greater than 50 times the uncertainty level, see Figure 9(c)). Conversely, using the proposed PCA references, the differences remained very small with respect to 


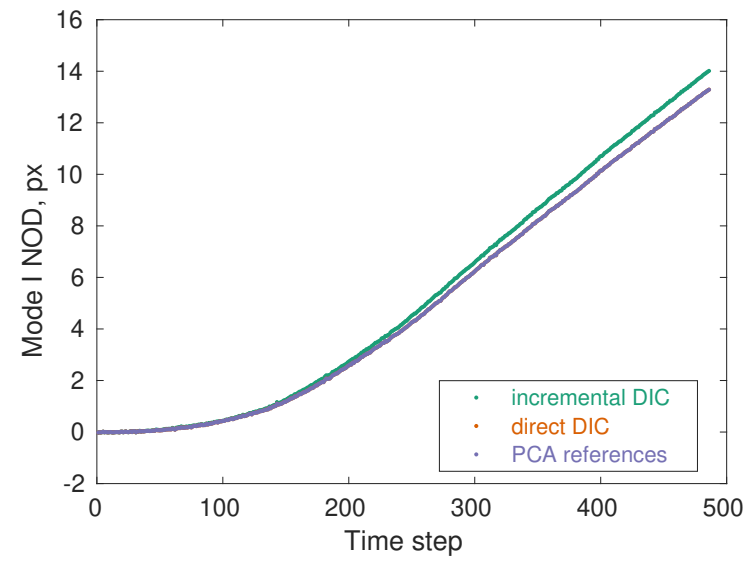

(a)

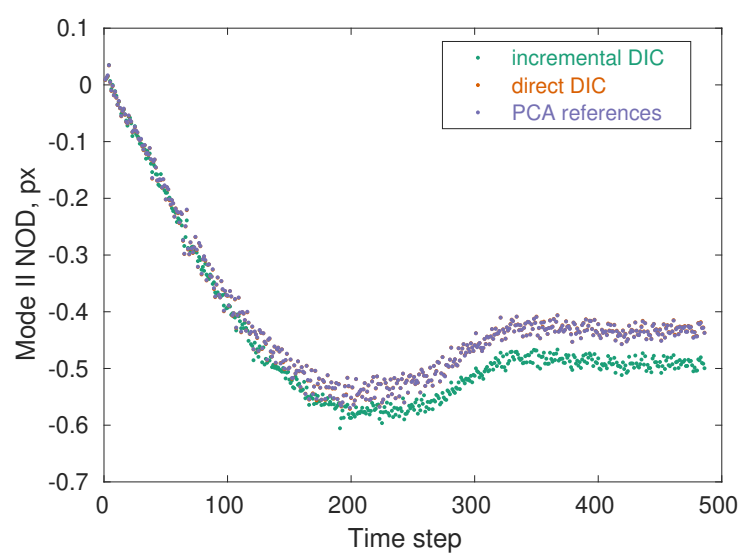

(b)

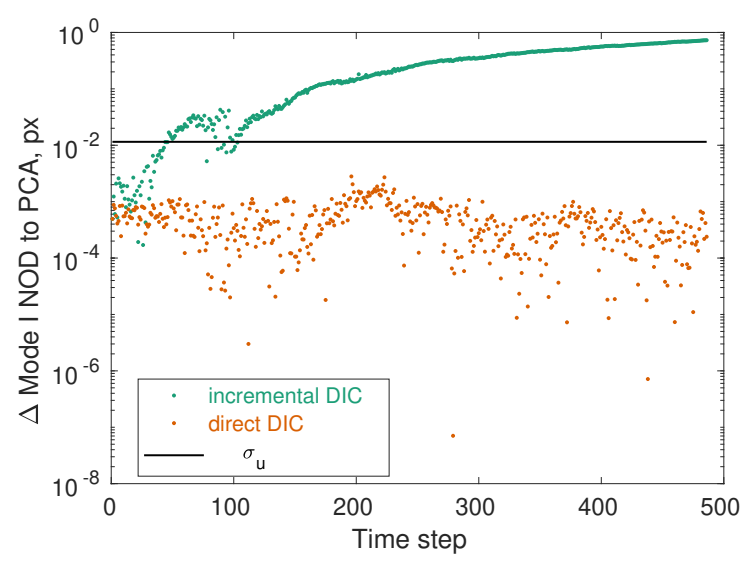

(c)

Figure 9: (a) Mode I and (b) mode II NODs for the room temperature WST (MZ1-S1450G). (c) Absolute difference of mode I NODs using PCA references and the other cases. The black solid line corresponds to the standard displacement uncertainty $\sigma_{u}$. Note that the results for PCA references appear to coincide with those of direct DIC in sub-figures (a) and (b). Their differences, shown in sub-figure (c) for mode I NOD, are very small compared to their magnitude.

The fracture energy $\Gamma_{c}^{N O D}$ was calculated for the three mode I NOD estimates reported in Figure 9. The incremental 215 approach led to $117 \mathrm{~J} / \mathrm{m}^{2}$, while the direct and PCA-based analyses yielded $111 \mathrm{~J} / \mathrm{m}^{2}$. The difference of about $6 \%$ was 216 mainly related to the drift observed in the incremental approach in comparison with the other two analyses (Figure 9(a)), 217 which is due to the propagation of uncertainties of the former.

\subsection{High temperature experiments}

The following analyses deal with four high temperature WSTs in which pattern extinctions occurred. More infor- 220 mation about these experiments can be found in Ref. [33]. 


\subsubsection{Test MZ2-S1400}

First, the PCA results for the MZ2-S1400 sample are shown in Figure 10. In that case, the fourth eigenvalue was 223 already less than $1 \%$ of the first one (Figure 10(a)). From the temporal modes (Figure 10(b)) it was concluded that most of the signal was comprised in the first two modes, which was also confirmed by the spatial modes (Figure 10(c-e)). The first mode, similar to the previous case, led to a quasi-constant reference image. The second mode helped to correct for vertical illumination gradients, which are believed to be due to shadows in the top region considering the direction of applied displacements in the experiment (Figure 6(c,d)). The dots seen in the third spatial mode, although not used in the truncation, were in positions very similar to the darker dots in the first spatial mode. They may be related to fluctuating light intensity during the experiment. Let us note that direct DIC converged with no need for updating the reference image, thereby indicating that speckle extinction remained limited.

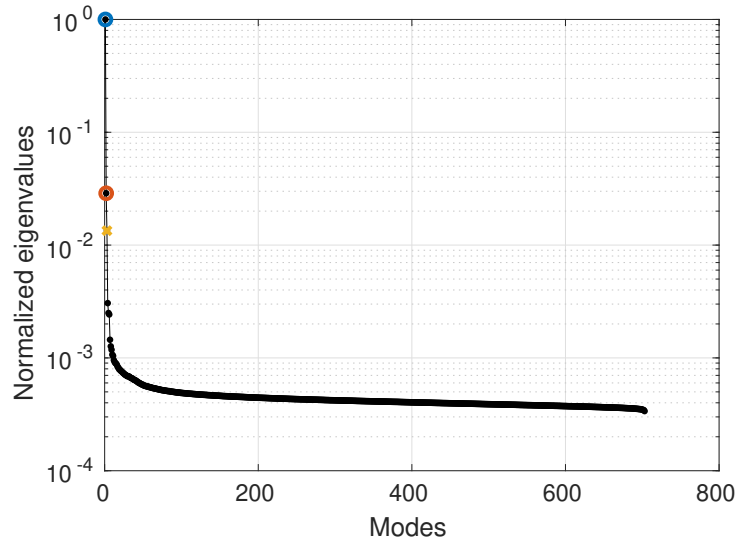

(a)

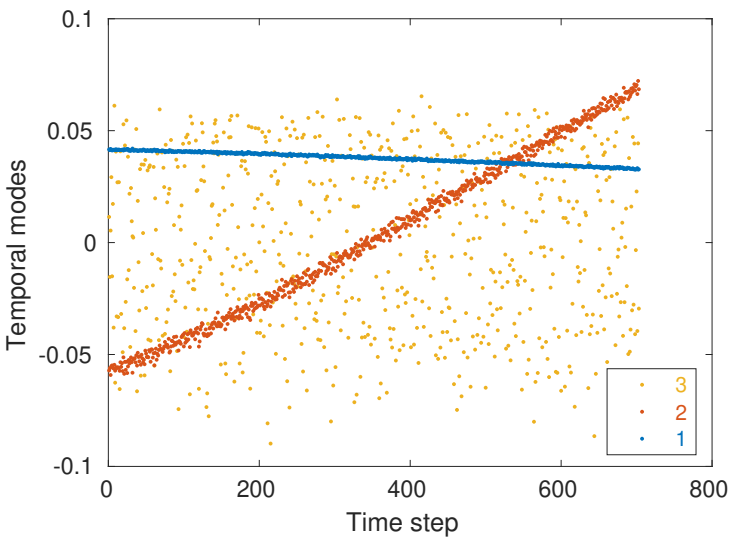

(b)

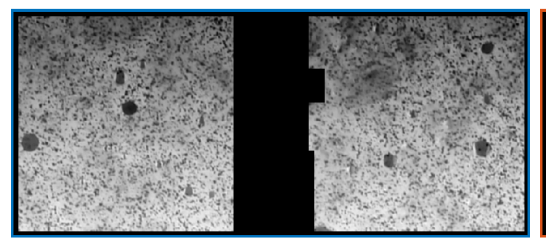

(c)

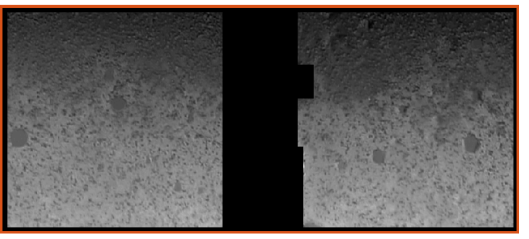

(d)

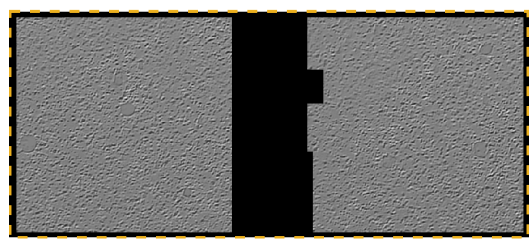

(e)

Figure 10: (a) Normalized eigenvalues of SVD of $\widetilde{g}$ for the first WST at $600^{\circ} \mathrm{C}$ (MZ2-S1400). (b) First three temporal modes and (c-e) corresponding spatial modes of the two ROls used for NOD measurements (Figure 5)

The results obtained with Algorithms 1 and 2 are shown in Figure 11. Two terms were sufficient for the truncation to bring displacement updates less than $\sigma_{u}=14.5 \mathrm{cpx}$ for every analyzed image. It is worth noting that only few points remained above $\sigma_{u}$ when only one mode was used, meaning that the first term brought most of the information 
(as expected). The average gray level residuals were significantly reduced, although they increased for the first 200 235 images when only one mode was accounted for. When the second mode was added, the residuals remained constant around $1 \%$. Similar to the room temperature case, Algorithm 2 converged in one single iteration.

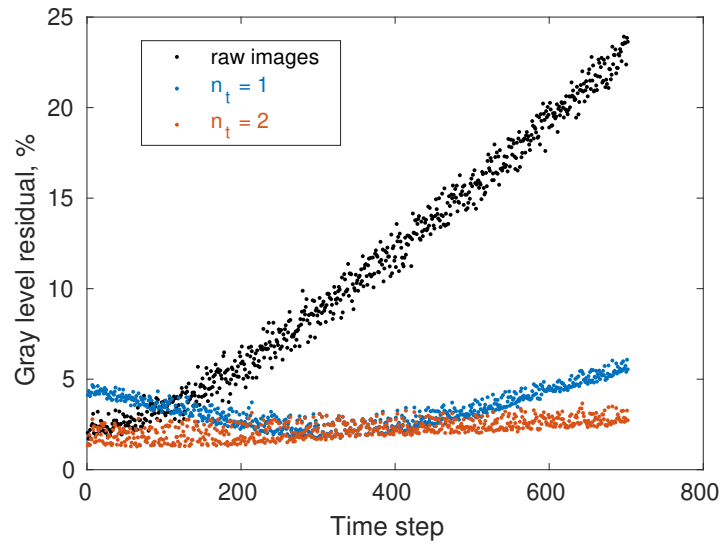

(a)

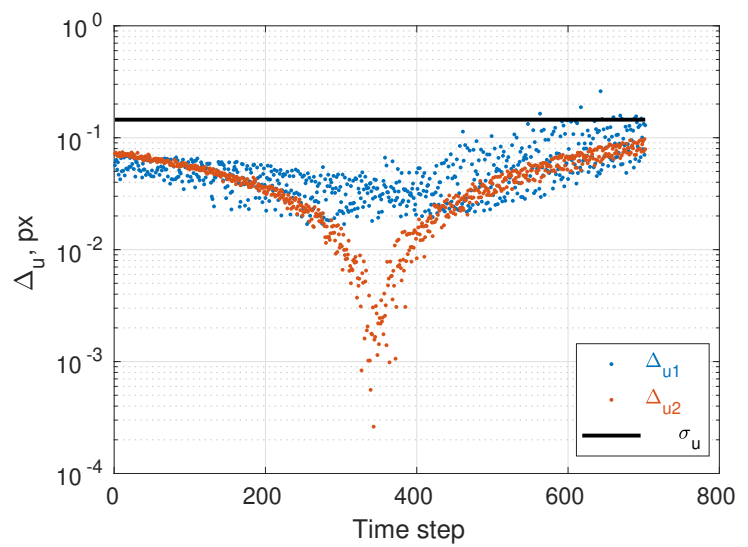

(b)

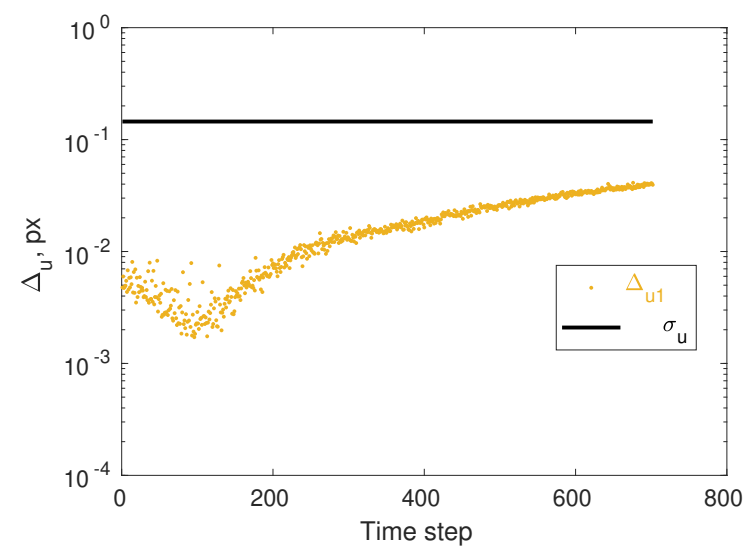

(c)

Figure 11: (a) Normalized RMS residuals for the first WST at $600^{\circ} \mathrm{C}$ (MZ2-S1400) using PCA-based references $\varphi^{1}$. (b) RMS difference between displacements for each tested truncation (Algorithm 1) and (c) for the proposed brightness correction (Algorithm 2). The standard displacement uncertainty $\sigma_{u}$ in solid black line

For this case, very small differences were observed in the measured NODs (Figure 12). As a consequence, very 238 small deviations occurred in $\Gamma_{c}^{N O D}$, which was equal to 187,191 and $192 \mathrm{~J} / \mathrm{m}^{2}$ for the incremental, direct and PCAbased analyses, respectively. Such fluctuation levels are even lower than those in the room temperature test. 


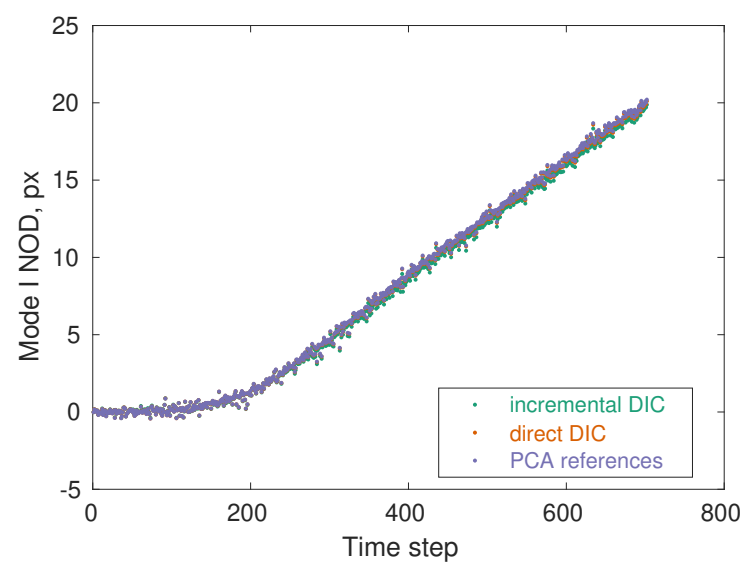

(a)

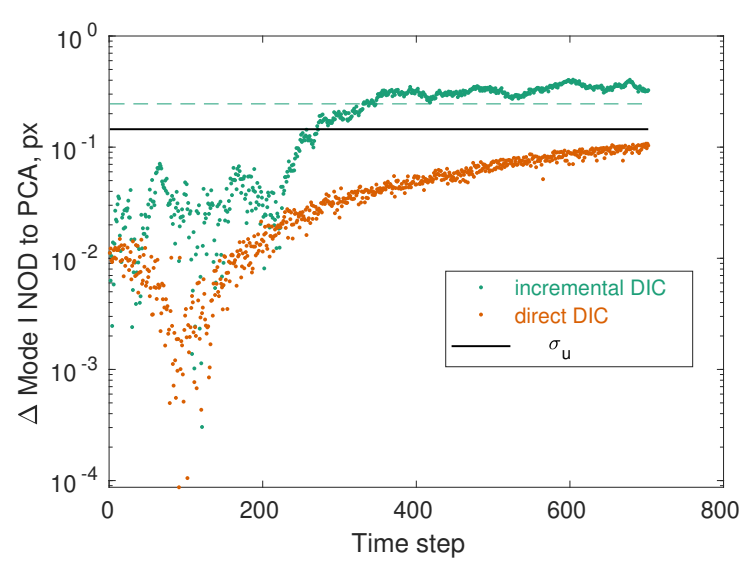

(b)

Figure 12: (a) Mode I NOD for the first WST at $600^{\circ} \mathrm{C}$ (MZ2-S1400). (b) Absolute difference of mode I NOD using PCA references and the other cases

\subsubsection{Test MZ3-S1400G}

The results of SVD for test MZ3-SG1400G are shown in Figure 13. Apart from the first and second eigenvalues, all the others were at least two orders of magnitude lower than the first one (Figure 13(a)). The first temporal mode was again quasi-constant (Figure 13(b)). It is interesting to note that this case was the first that was cyclic, and the cycles are seen in the second temporal mode. This feature reinforces the hypothesis of the second mode being related to how 245 the applied vertical displacement changed the shadows that appear in the top regions (Figure 6). The speckle pattern is better seen in the first spatial mode (Figure 13(c)), while the second one comprises a vertical gradient (Figure 13(d)) due to illumination variations along the vertical axis. 


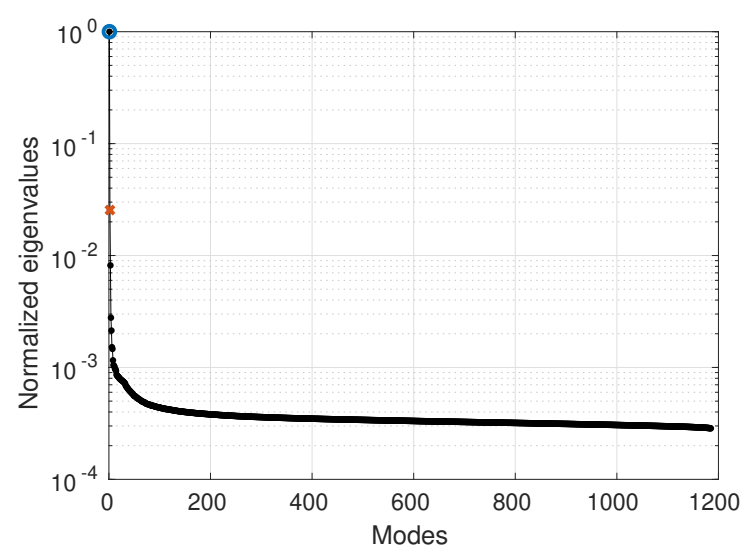

(a)

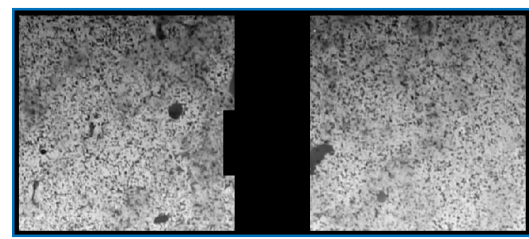

(c)

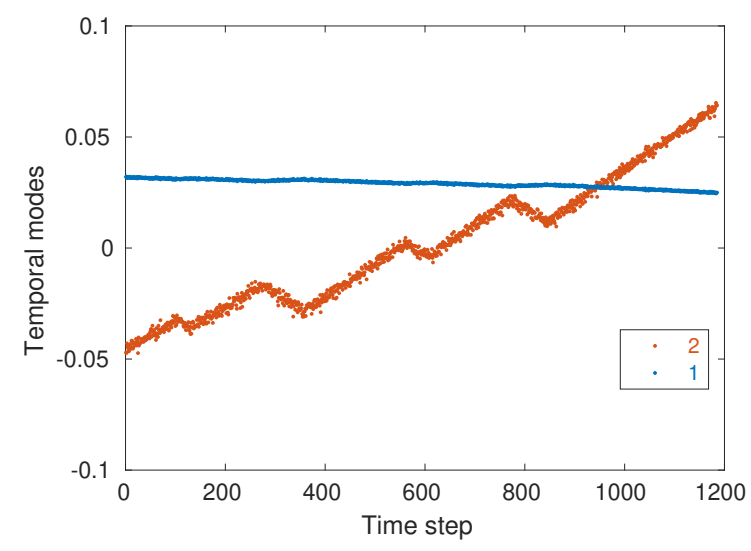

(b)

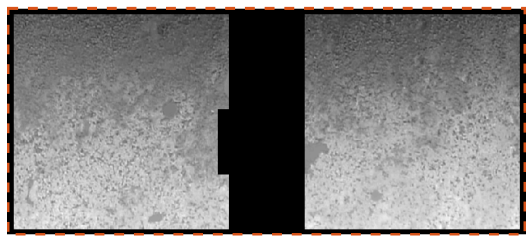

(d)

Figure 13: (a) Normalized eigenvalues of SVD of $\widetilde{g}$ for the second WST at $600^{\circ} \mathrm{C}$ (MZ3-S1400G). (b) First two temporal modes and (c-d) corresponding spatial modes of the two ROls used for NOD measurements (Figure 5)

The truncation with only the first term significantly reduced the average gray level residuals (Figure 14(a)), although they increased for the first 100 images. A single iteration was sufficient to get displacement updates less than $\sigma_{u}=\mathbf{2 5 0}$ $16.5 \mathrm{cpx}$ for the truncation (Figure 14(b)) and for the PCA correction (Figure 14(c)) step. Although the second temporal 251 mode indicated that the second mode may contain relevant information, these results show that by using only the first $\mathbf{2 5 2}$ mode the displacements difference was already less than the uncertainty level. 


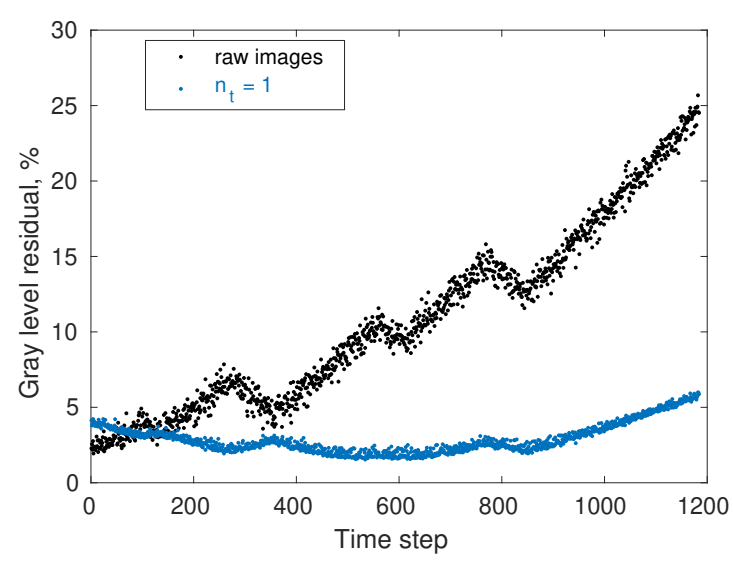

(a)

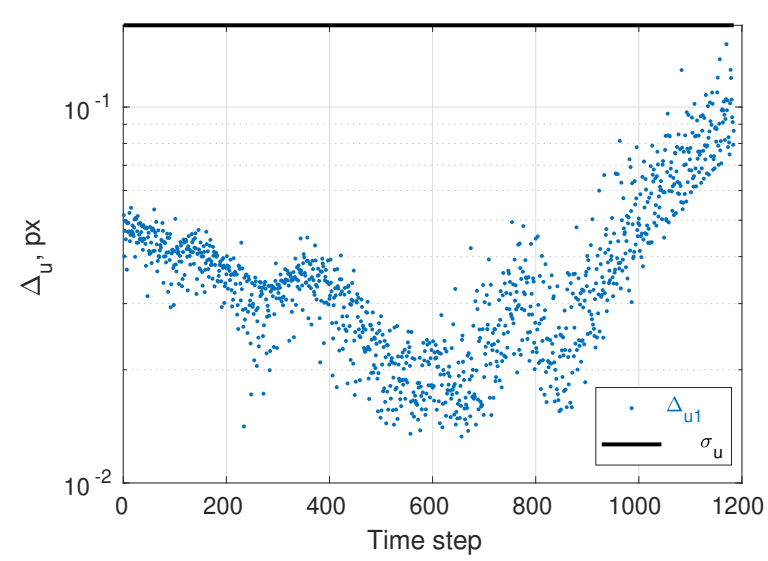

(b)

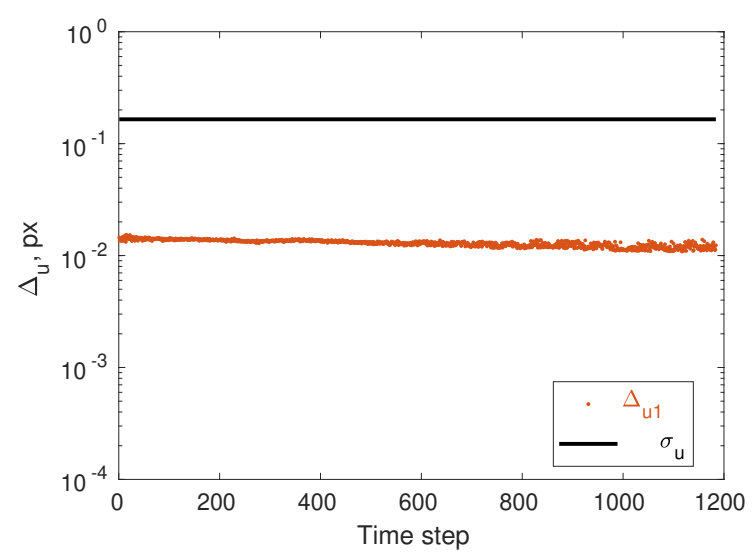

(c)

Figure 14: (a) Normalized RMS residuals for the second WST at $600^{\circ} \mathrm{C}$ (MZ3-S1400G) using PCA-based references $\varphi^{1}$. (b) RMS difference between displacements for each tested truncation (Algorithm 1) and (c) for the proposed PCA correction (Algorithm 2). The standard displacement uncertainty $\sigma_{u}$ is shown in solid black line

The NODs calculated for MZ3-S1450G for the three approaches were virtually identical (Figure 15). The in- 254 cremental case provided $\Gamma_{c}^{N O D}=158 \mathrm{~J} / \mathrm{m}^{2}$, while both direct and PCA-based analyses resulted in $155 \mathrm{~J} / \mathrm{m}^{2}$ with a 255 difference less than 2\%. Although the PCA corrections enabled very small gray level residuals to be reached, no 256 significant difference was seen in the NODs when compared to regular DIC results. 


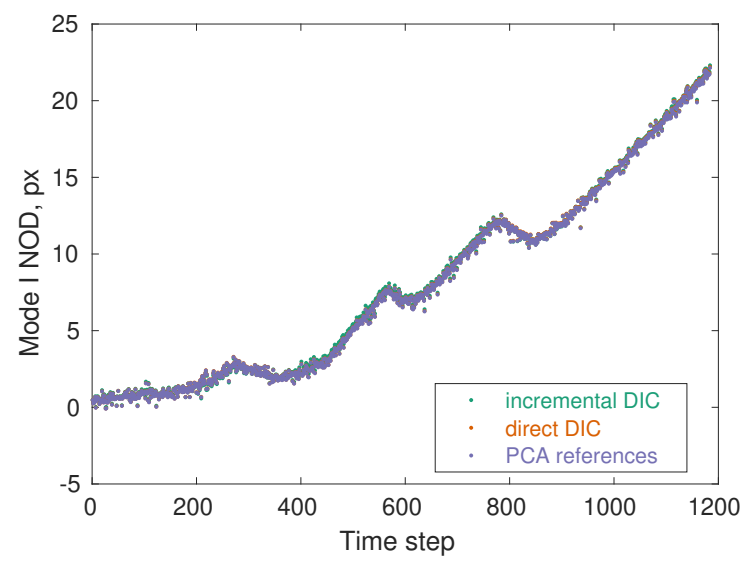

(a)

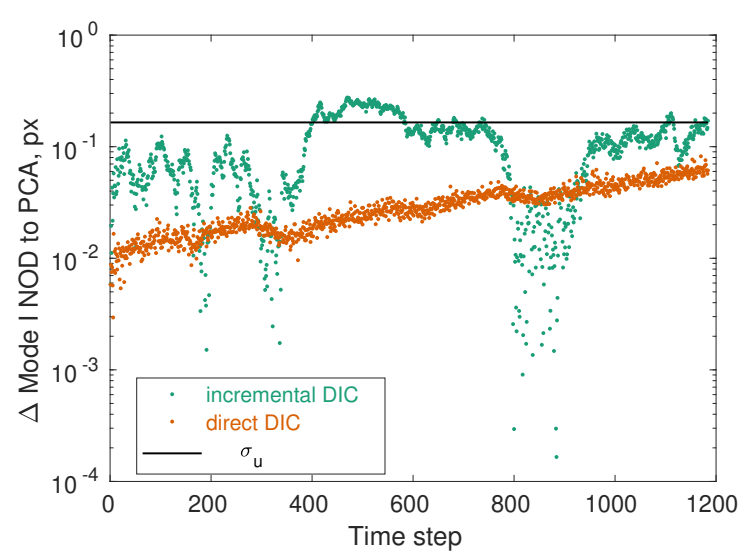

(b)

Figure 15: (a) Mode I NOD for the second $600^{\circ} \mathrm{C}$ WST (MZ3-S1400G). (b) Absolute difference of mode I NOD using PCA references and the other cases

\subsubsection{Test MZ4-S1450}

WST MZ4-S1450 is now analyzed. For this test and the last WST, direct DIC did not converge and thus references

were updated in the quasi-direct framework. It was the first experiment to require more than two terms in the truncation procedure. Although the first three eigenvalues were distributed similarly to the previous cases (Figure 16(a)), their temporal components (Figure 16(b)) exhibited considerable differences. The first two were similar, with one quasi- 262 constant and a second one that followed the loading history. However, some signal was still seen in the third mode, 263 which had less random fluctuations than in the previous experiments. The major pattern contribution is observed in the $\mathbf{2 6 4}$ first spatial mode (Figure 16(c)), while a vertical gradient is present in the second one (Figure 16(d)). Some contrast 265 is still observed in the third (Figure 16(e)) and even in the fourth (Figure 16(f)) modes. They are believed to be related 266 to displacements that could not be fully corrected with the chosen kinematic basis (e.g., due to heat haze). 


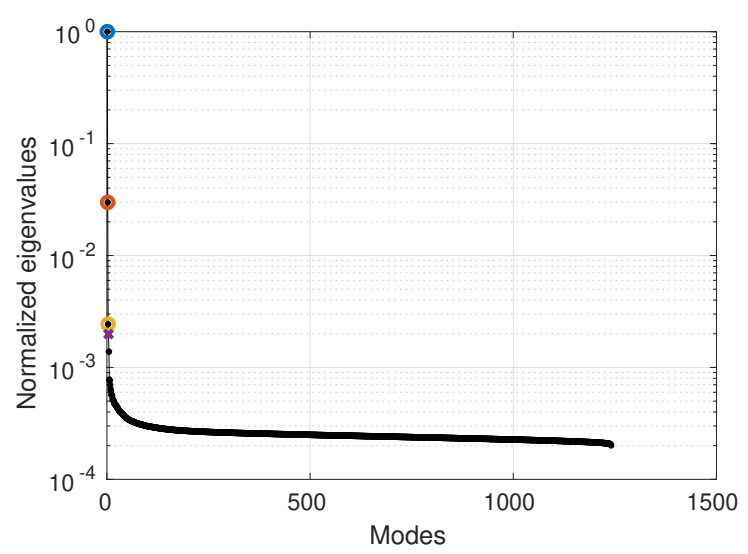

(a)

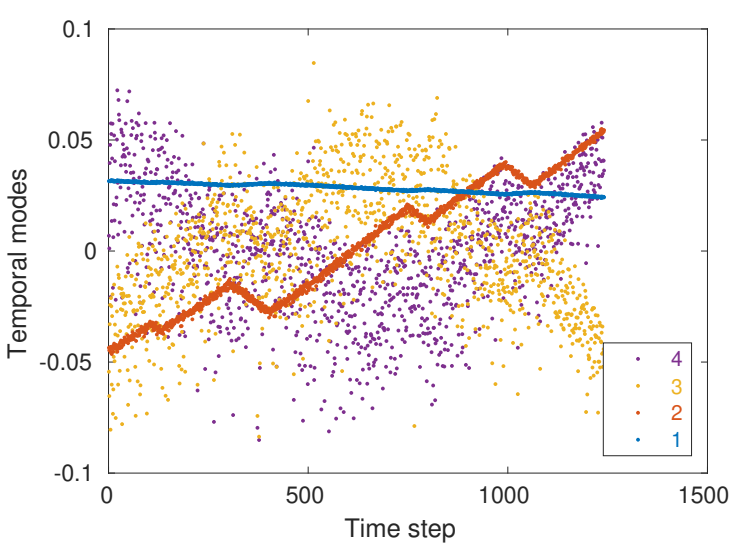

(b)

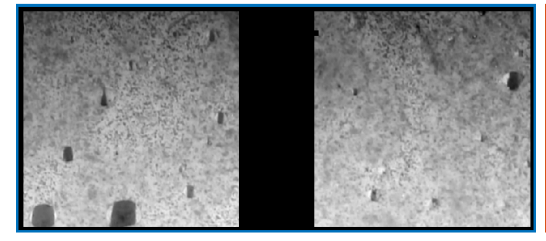

(c)

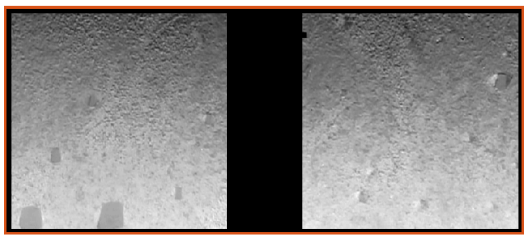

(d)

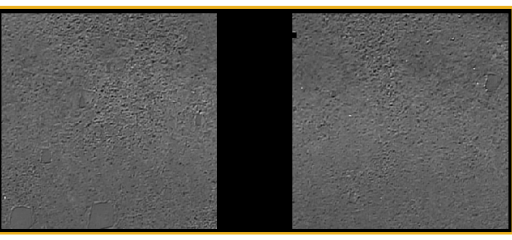

(e)

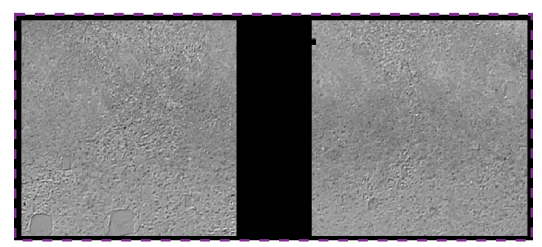

(f)

Figure 16: (a) Normalized eigenvalues of SVD of $\widetilde{g}$ for the third WST at $600^{\circ} \mathrm{C}$ (MZ4-S1450). (b) First four temporal modes and (c-f) corresponding spatial modes of the two ROIs used for NOD measurements (Figure 5)

The contribution of each of the three modes in reducing the gray level residual is shown in Figure 17(a). The first 268 term increased the residuals at the beginning and reduced them for the remainder of the experiment. Adding the second $\mathbf{2 6 9}$ term already flattened out considerably the residuals, which became almost indistinguishable when three modes were 270 considered in the truncation procedure. A very flat residual was obtained, always less than $1 \%$, which is very low. 271 The difference in displacements during the truncation procedure (Figure 17(b)) also shows how with one or two terms 272 almost the whole curve remained above the selected threshold $\sigma_{u}=14.0 \mathrm{cpx}$ apart from the beginning and end of the 
test. When the third mode was added, it helped to lower the residual overall. It is worth noting that only one reference needed to be updated in quasi-direct DIC, which was close to the time step when $\Delta_{u}$ was greater than $\sigma_{u}$ when one or two terms were used. Once the truncation was achieved, the brightness correction converged in one single iteration (Figure 17(c)), thereby proving the robustness of the methodology.

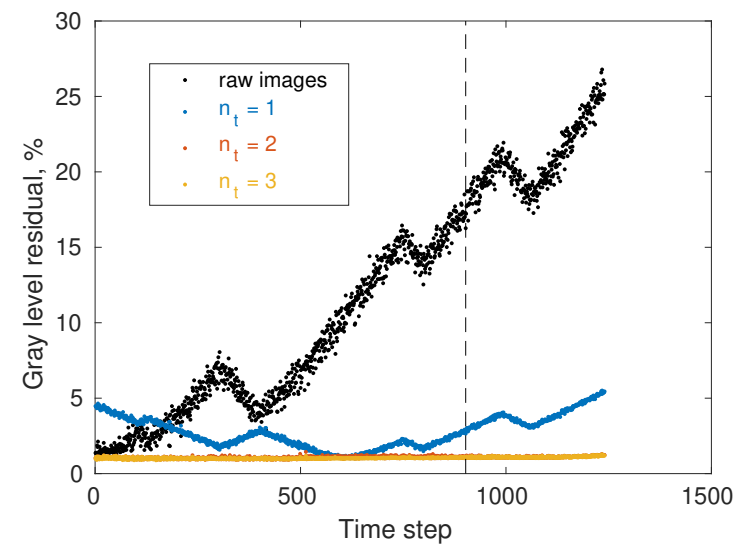

(a)

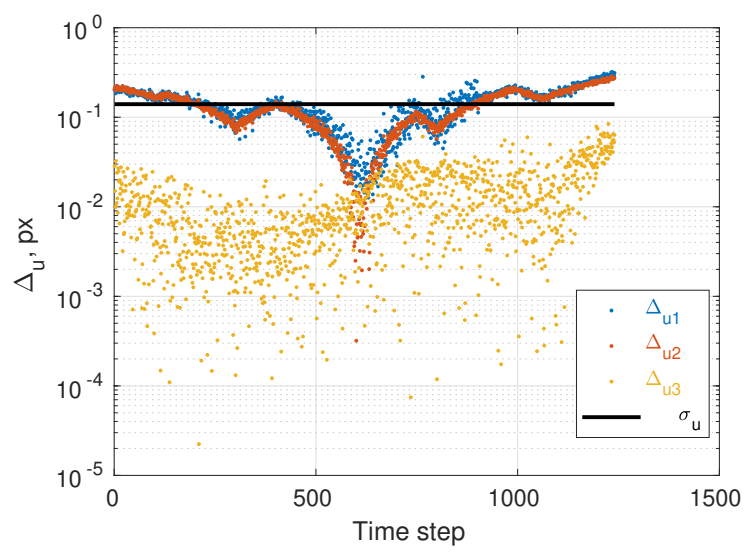

(b)

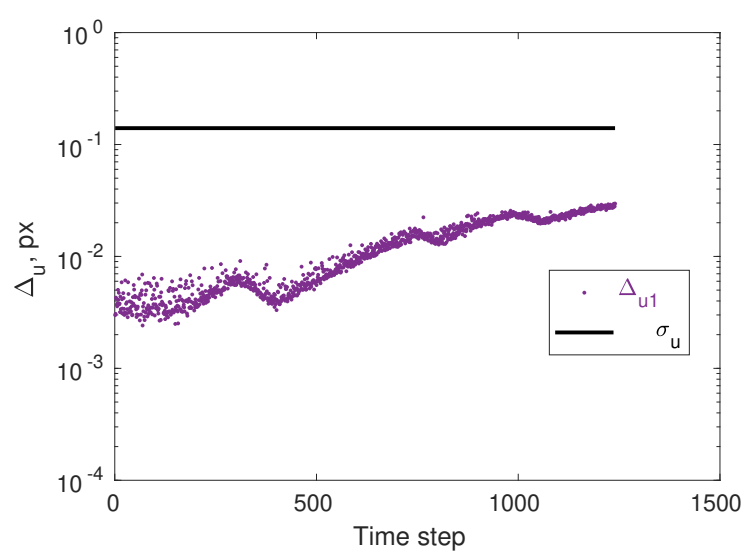

(c)

Figure 17: (a) Normalized RMS residuals for the third WST at $600^{\circ} \mathrm{C}$ (MZ4-S1450) using PCA-based references. The reference update for quasi-direct DIC is depicted with a dashed vertical line. (b) RMS difference between displacements for each tested truncation (Algorithm 1) and (c) for the proposed brightness correction (Algorithm 2). The standard displacement uncertainty $\sigma_{u}$ is shown in solid black line

The NOD measurements for WST MZ4-S1450 are shown in Figure 18. Their differences were very small, and 278 provided very close estimates for $\Gamma_{c}^{N O D}$ (i.e., 229 for incremental DIC, and $227 \mathrm{~J} / \mathrm{m}^{2}$ for quasi-direct DIC as well as 279 PCA references). The present case shows that if direct DIC results are not accessible, the incremental approach may 280 provide acceptable results as the brightness variations remained incrementally small, even if care should be taken (see 
drifts in the first analyzed experiment).

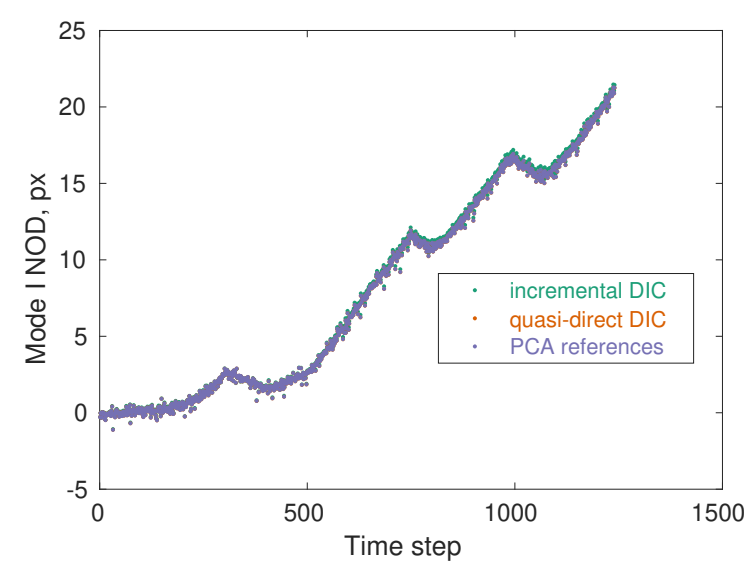

(a)

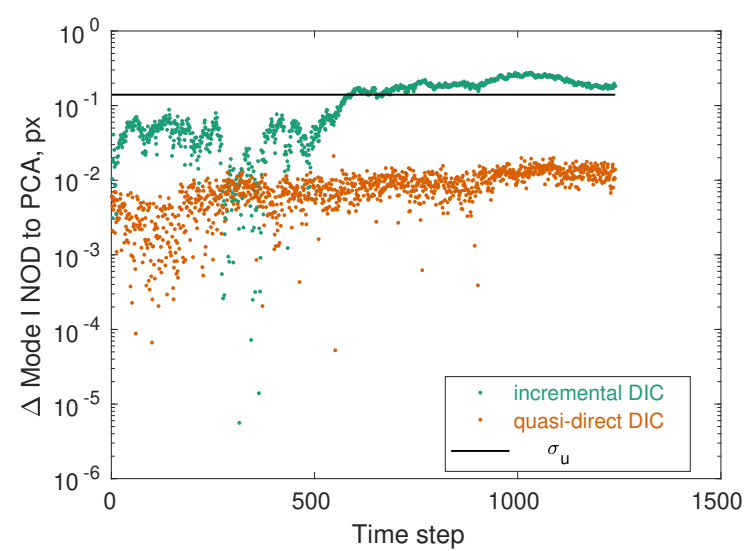

(b)

Figure 18: (a) Mode I NOD for the third $600^{\circ} \mathrm{C}$ test (MZ4-S1450). (b) Absolute difference of mode I NOD using PCA references and the other cases

\subsubsection{Test MZ5-S1450G}

From all the analyzed WST tests, MZ5-S1450G was expected to be the most challenging case (Figure 6). Since direct DIC did not converge, incremental followed by quasi-direct DIC was carried out and the results of SVD are shown in Figure 19. This is a case where more modes had normalized eigenvalues close to $10^{-2}$ (Figure 19(a)). The first two temporal modes show trends close to those seen previously (Figure 19(b)), and similar spatial modes (Figure 19(cd)). In that case, Algorithm 1 proposed a truncation with 6 terms. From the third mode on (Figures 19(e-f)), some unresolved displacements are observed in different regions. This observation reinforces the hypothesis that modes above the first two may include components of displacements that could not be captured by the chosen kinematic basis (e.g., artifactual displacements caused by heat haze or speckle extinction). From the analyzed WSTs, these higher order modes appear to become more important with the duration of the experiment (i.e., more time frames were affected by such issues). 


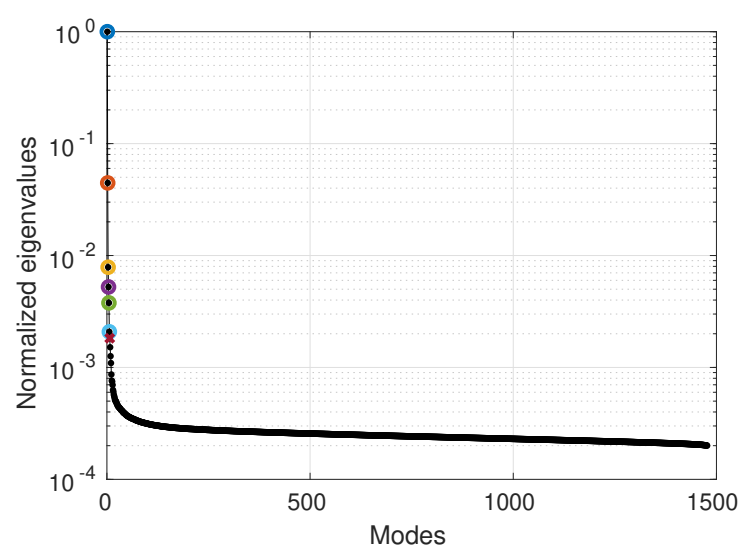

(a)

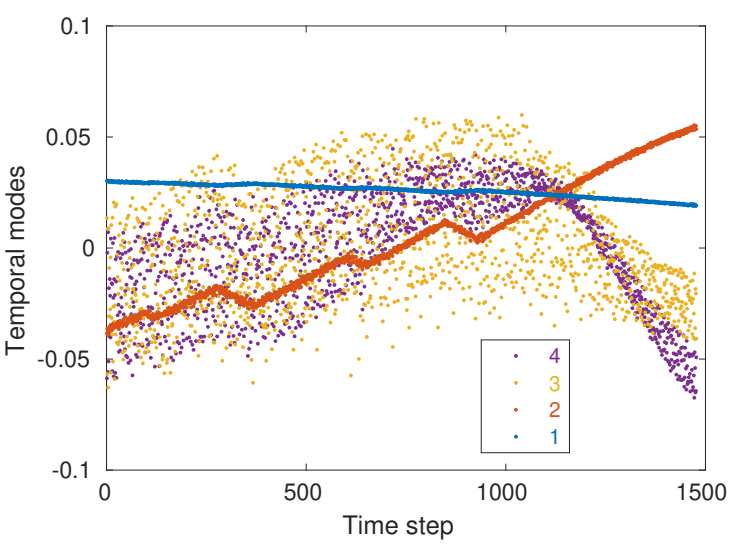

(b)

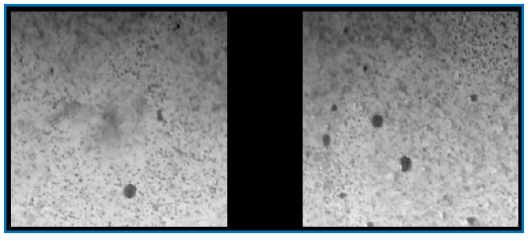

(c)

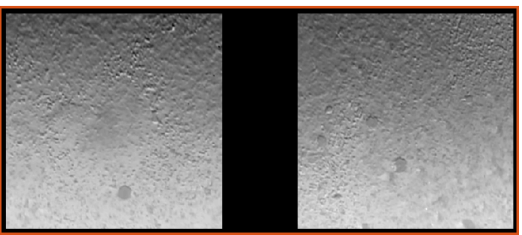

(d)

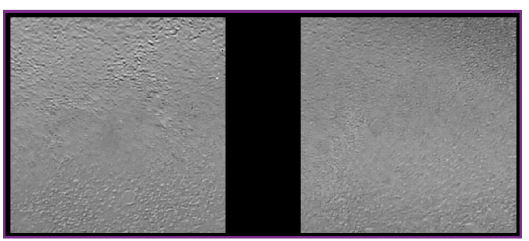

(f)

\section{)}

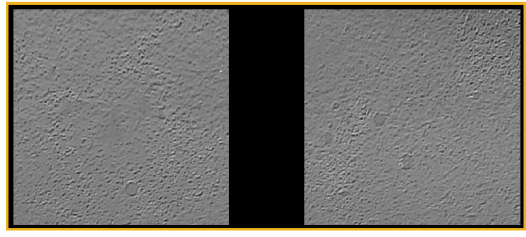

(e)

Figure 19: (a) Normalized eigenvalues of SVD of $\widetilde{g}$ for the fourth WST at $600^{\circ} \mathrm{C}$ (MZ4-S1450G). (b) First four temporal modes and (c-f) corresponding spatial modes of the two ROls used for NOD measurements (Figure 5)

The results of Algorithms 1 and 2 are shown in Figure 20. Quasi-direct DIC needed to update the reference state

four times. If no brightness corrections were accounted for, the gray level residuals went up to $40 \%$ of the dynamic

range. It was reduced to about $5 \%$ using only the first mode (Figure 20(a)). The residual reduction by adding more terms was less noticeable and became very small (i.e., less than $2 \%$ for every time step). By checking the displacement

differences $\Delta_{u}$ for each truncation (Figure 20(b)), the third and fourth terms improved the kinematics especially for the second part of the experiment but remained very close to $\sigma_{u}$ (being above it for few time steps). Algorithm 2 again 
converged in one single iteration (Figure 20(c)).

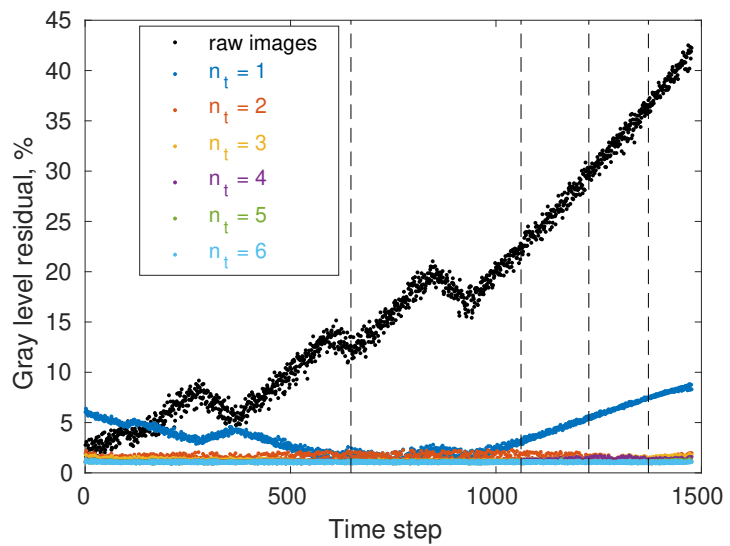

(a)

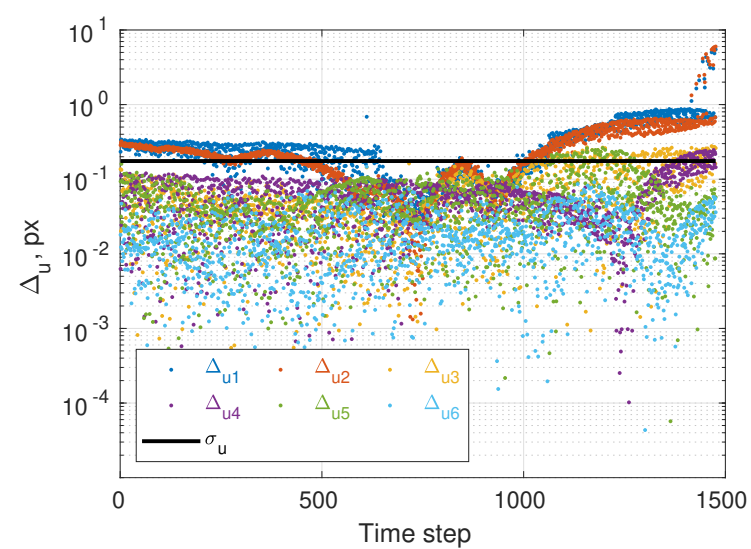

(b)

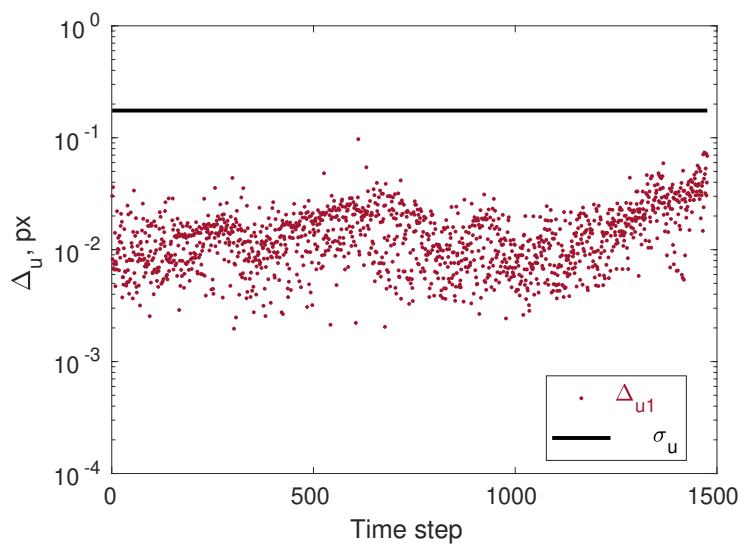

(c)

Figure 20: (a) Normalized RMS residuals for the fourth WST at $600^{\circ} \mathrm{C}$ (MZ5-S1450G) using PCA-based references $\varphi^{1}$. The four reference updated in quasi-direct DIC are shown in vertical dashed lines. (b) RMS difference between displacements for each tested truncation (Algorithm 1) and (c) for the proposed brightness correction (Algorithm 2). The standard displacement uncertainty $\sigma_{u}$ is shown in solid black line.

Last, the NODs were evaluated and are shown in Figure 21. Again, similar trends are observed. The difference in mode I NOD from PCA to incremental DIC was small at the beginning of the test but increased up to the pixel level while from PCA and quasi-direct DIC remained very close (Figure 21(b)). If only the incremental case were considered, the fracture energy $\Gamma_{N O D}^{c}$ was equal to $201 \mathrm{~J} / \mathrm{m}^{2}$, in comparison to $206 \mathrm{~J} / \mathrm{m}^{2}$ for quasi-direct DIC and PCA cases. Therefore, even in this troublesome case, using a robust kinematic basis can help to regularize and obtain reliable results. 


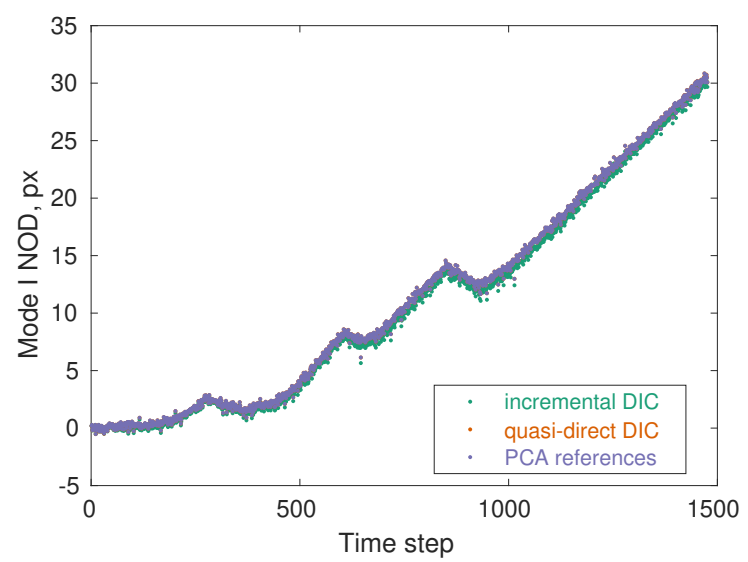

(a)

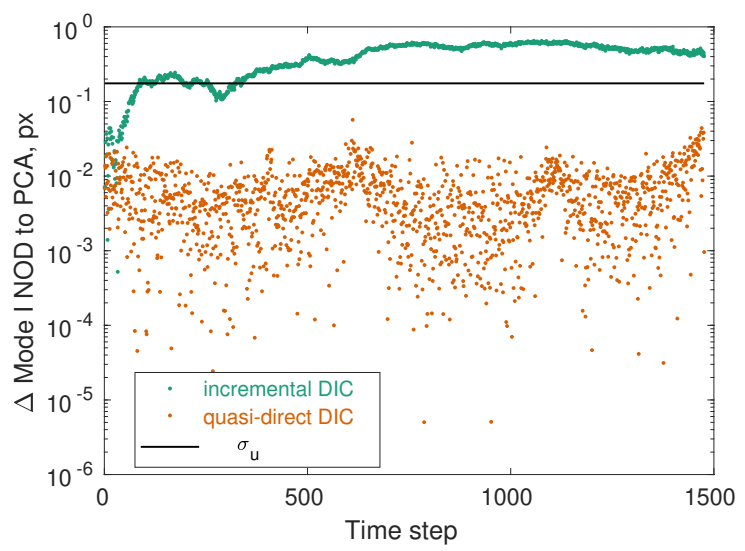

(b)

Figure 21: (a) Mode I NOD for the fourth $600^{\circ} \mathrm{C}$ case (MZ5-S1450G). (b) Absolute difference of mode I NOD using PCA references and the otehr cases

\section{Dilatometry test}

The present section addresses the dilatometry experiment that was used to motivate the present framework. Given all the initial discussions of Section 2.1, this case was deemed very difficult. The first three normalized eigenvalues spanned over two orders of magnitude (Figure 22(a)). The first temporal mode (Figure 22(b)) was almost constant, 310 which is similar to the previously analyzed WSTs. The first spatial mode (Figure 22(c)) shows the initial pattern with 311 porosities and some contrast. The second temporal mode was very interesting since its trend was similar to that of $\mathbf{3 1 2}$ the gray level residuals (Figure 4(b)). Moreover, it started off with a higher value and decreased considerably, which is consistent with the extinction of the pattern. Such evidence is confirmed by the second spatial mode shown in $\mathbf{3 1 4}$ Figure 4(d) where the speckle pattern is clearly visible and dominant. The black speckle dots were also seen in the $\mathbf{3 1 5}$ third spatial mode while the fourth shows darker regions with very few of the finer speckle. For instance, the third 316 temporal mode was mostly concentrated in the transition regime of the second one. Their combination allowed for a $\mathbf{3 1 7}$ refined description of such changes. 


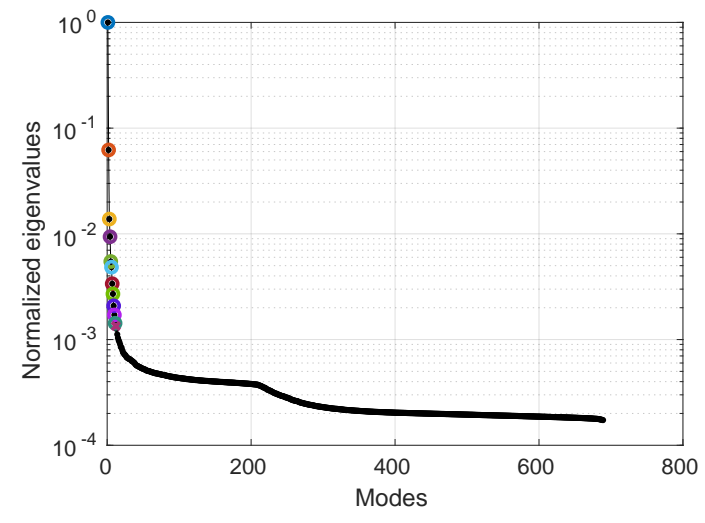

(a)

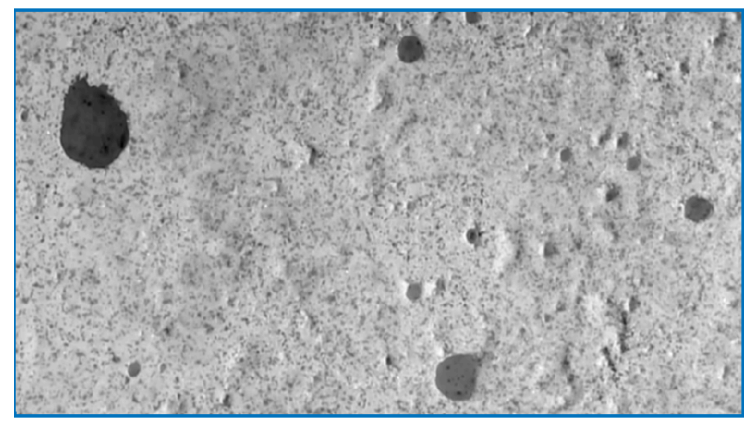

(c)

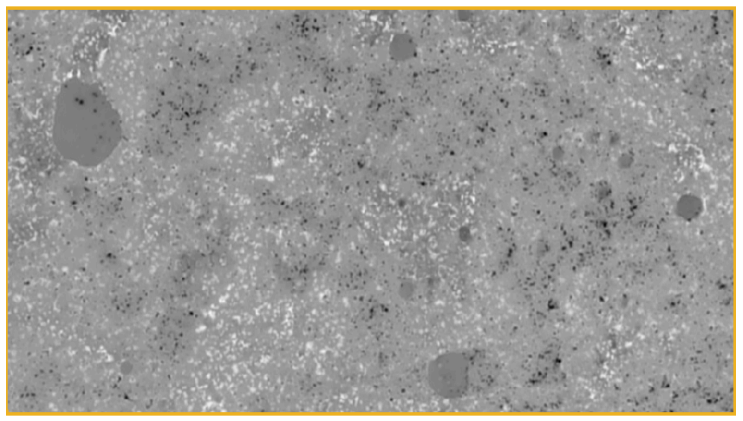

(e)

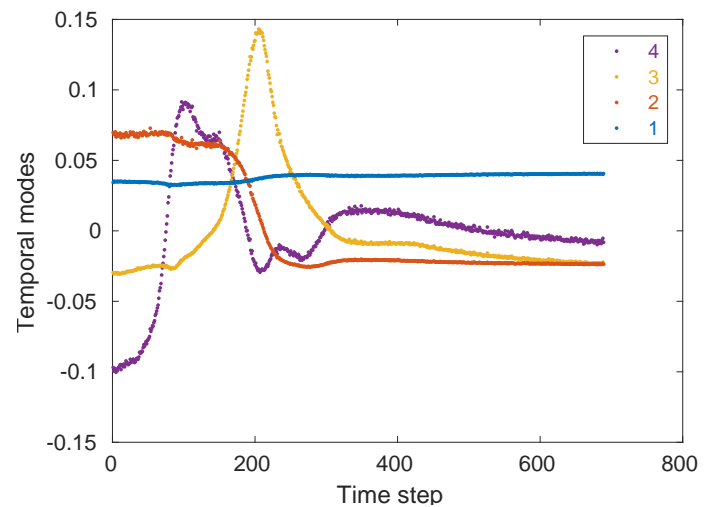

(b)

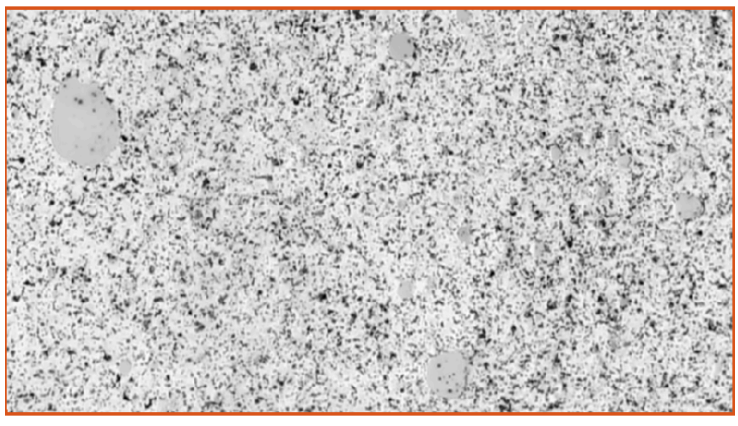

(d)

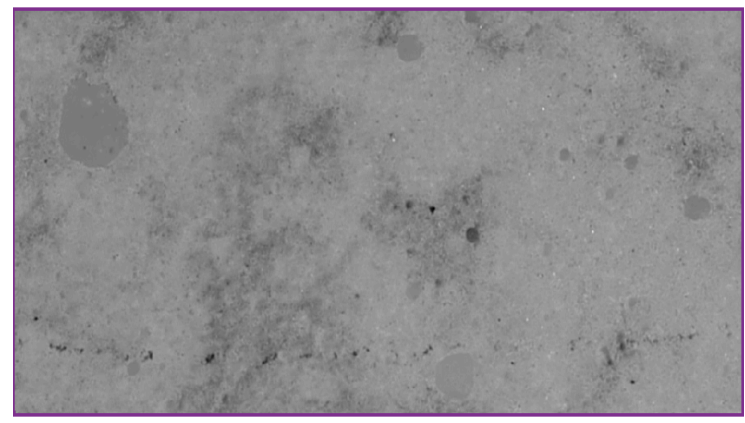

(f)

Figure 22: (a) Normalized eigenvalues for the SVD decomposition of $\tilde{g}$ corresponding to the dilatometry test. (b) First four temporal modes and (c-f) corresponding spatial modes

The normalized gray level residuals (wrt. the dynamic range of $f\left(\mathbf{x}, t_{0}\right)$ ) are shown in Figure 23(a). For every 
tions). It also shows how adding each new mode helps reducing the residuals. Using four terms was the first truncation that led to smaller residuals than the reference case for every time step with small fluctuations but always less than $2 \%$, which was already less than the gray level residuals calculated for the 100 reference images before the experiment.

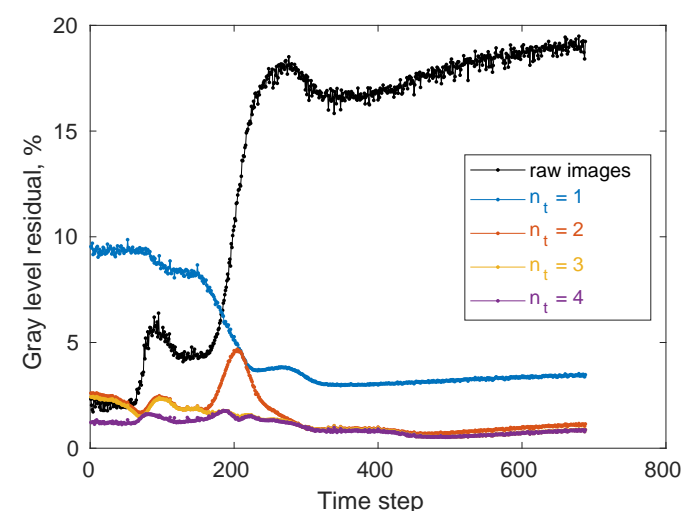

(a)

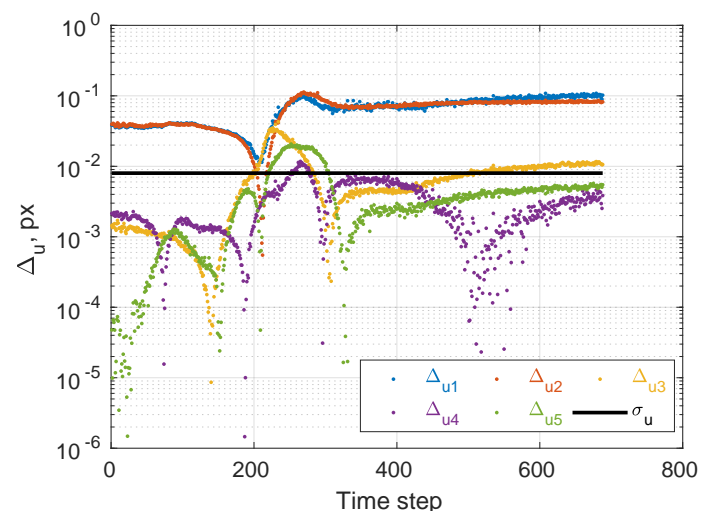

(b)

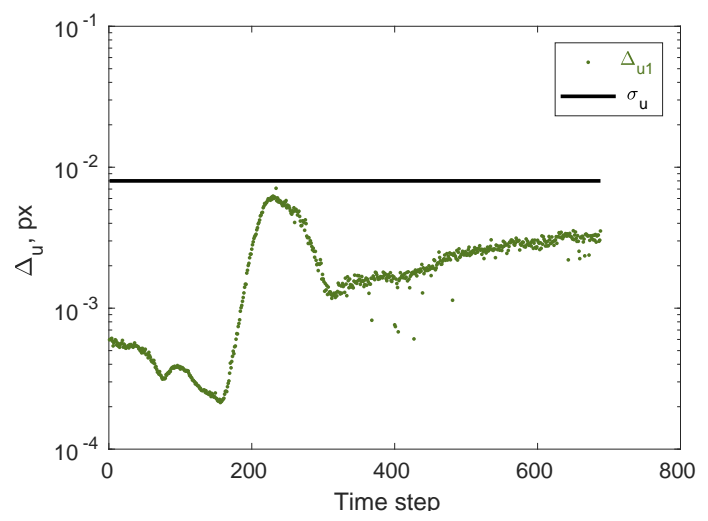

(c)

Figure 23: (a) Normalized RMS residuals for the preliminary analysis and for the case using PCA-based references. (b) RMS difference between displacement for each tested truncation (Algorithm 1). (c) RMS displacement difference for the first step of Algorithm 2. The standard displacement uncertainty $\sigma_{u}$ is shown with a solid black line

For each DIC calculation performed in the truncation step, the RMS displacement difference wrt. the previous step is shown in Figure 23(b). It is worth noting that using $n_{t}=4$ modes kept only 24 steps with displacement 325 residuals above the threshold (Figure 23(c)), between time steps 250 and 280, reduced from 264 images above the same threshold when $n_{t}=3$ was considered. Further, adding the fifth term increased to 84 time steps, and then to 324 when the sixth was also accounted for. As written in Algorithm 1, $\Delta_{u}$ of this difference is less than the standard displacement uncertainty $\sigma_{u}=0.8 \mathrm{cpx}$ only when the first 11 modes were considered. However, no studied WST exhibited such trend of increasing average $\Delta_{u}$ when more modes were added, nor an increase of the number of steps 
above the threshold. Therefore, the chosen number of terms for the truncation was $n_{t}=4$, which, once selected, 331 allowed Algorithm 2 to be run. It converged for a single iteration (Figure 23(c)). Even though the present case was 332 very challenging, there was no need to update the truncated basis.

In the dilatometry experiment, the main goal was to obtain a first order estimate of the coefficient of thermal $\mathbf{3 3 4}$ expansion (CTE) for the tested material. The dilatational strain $\epsilon_{t h}$ was estimated as the mean eigen strain $\left(\epsilon_{1}+\epsilon_{2}\right) / 2.335$ The dilatational strains are compared between the initial DIC calculation (with no correction) and the final converged 336 state in Figure 24(a). In this material, since no phase transformation nor chemical reaction was expected to take place $\mathbf{3 3 7}$ in the investigated temperature range [40], the strains should be in phase with the temperature history. Both approaches $\mathbf{3 3 8}$ led to dilatational strains that were consistent with the previous observation. Conversely, large fluctuations occurred 339 for the half difference of eigen strains around time step 300 (Figure 24(b)), which were artifactual and related to the 340 extinction of the speckle pattern together with heat haze effect due to high temperatures [33], since no anisotropy was $\mathbf{3 4 1}$ expected. The lack of pattern makes the oscillations at the end higher than those at similar temperatures during heating. 342 At the maximum temperature, their level was about one order of magnitude lower than the dilatational strain itself. $\mathbf{3 4 3}$ These measured strain differences were greater than the noise floor levels (i.e., $3 \times 10^{-5}$ ) estimated at room temperature. 344 Due to higher temperatures (about $600^{\circ} \mathrm{C}$ ) in this setup, it was expected that the noise floor level was about one order 345 of magnitude higher [33] due to heat haze effects caused by air motion inside the oven. If such factor were considered, 346 the noise floor would still be less than the reported fluctuations. Several images would need to be acquired at high $\mathbf{3 4 7}$ temperatures after speckle extinction to quantify their impact on displacement and strain uncertainties.

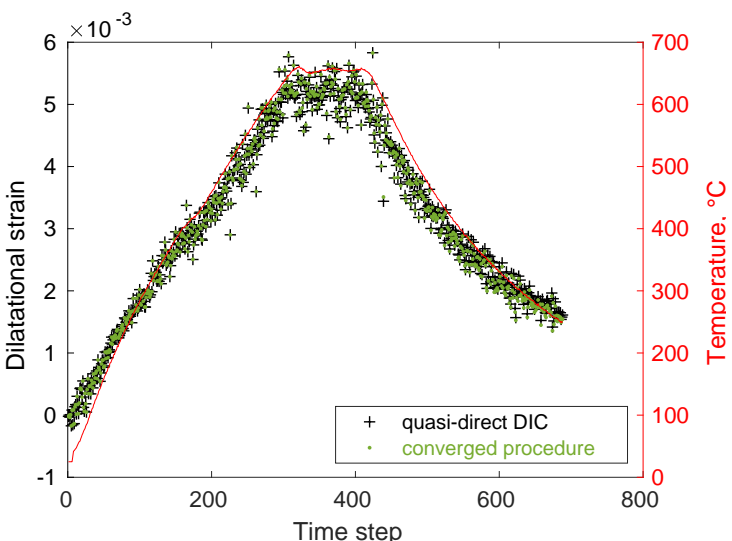

(a)

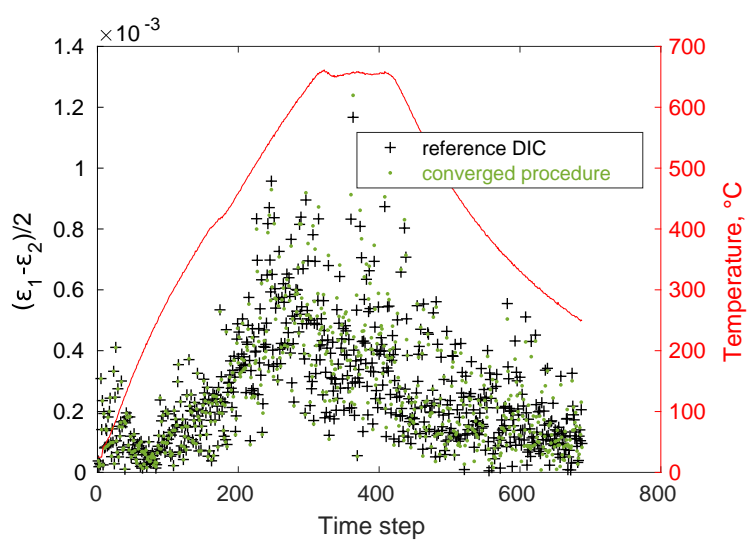

(b)

Figure 24: (a) Dilatational strain vs. time for DIC analyses with no brightness correction and for the last case after convergence. (b) Corresponding half difference of eigen strains. The temperature signals are shown in red

The slope of the dilatational strain vs. temperature response (Figure 25(a)) provided an estimate of the mean CTE 349 of the tested material. A value of $(7.6 \pm 0.08) \times 10^{-6}{ }^{\circ} \mathrm{C}^{-1}$ was obtained for incremental DIC, $(8.55 \pm 0.05) \times 10^{-6}{ }^{\circ} \mathrm{C}^{-1}$ 
for quasi-direct DIC, and $(8.65 \pm 0.05) \times 10^{-6}{ }^{\circ} \mathrm{C}^{-1}$ with the PCA correction. which is consistent with levels reported 351 for alumina [41] that comprises most of the matrix and for mullite-zirconia, that composes the aggregates in the studied $\mathbf{3 5 2}$ castable. Even if a higher scatter was observed for some time steps, it did not influence too much the evaluation of $\mathbf{3 5 3}$ the CTEs. The use of a reduced kinematic basis provided sufficient regularization to obtain these results even without $\mathbf{3 5 4}$ PCA correction since only the overall CTE was investigated.

To further probe the quality of the measurements, the half difference in eigen strains is reported in Figure 25(b) 356 for the two analyses. In the present case, it was expected that their difference should be as small as possible. The $\mathbf{3 5 7}$ PCA-based procedure led to lower overall differences even though they still were similar to the quasi-direct approach, 358 oscillating more at the point where the pattern disappeared. Last, for incremental DIC, there was a gradual drift, which 359 shows that even though the CTE evaluation was consistent, care should be exercised when using such approaches. If 360 direct analyses are possible, they should be preferred. Otherwise, it should be remembered that drifts may occur due to cumulative uncertainties and brightness changes.

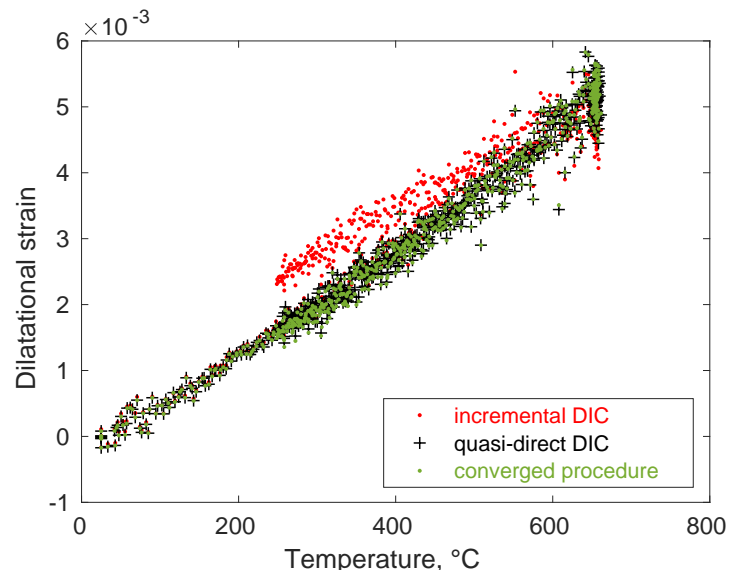

(a)

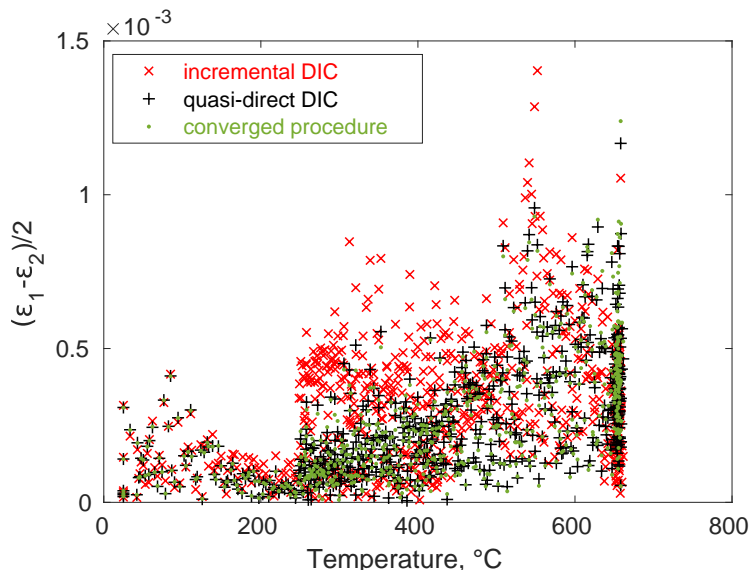

(b)

Figure 25: Dilatometry results showing the dilatational strain (a) and the half-difference between both eigen strains (b) as functions of temperature.

\section{Conclusion}

A procedure based on Principal Component Analysis (PCA) was introduced to build a set of reference images suited to improve DIC analyses in cases of pattern extinction. First, a stack of estimates for the reference state with initial (coarse) kinematic corrections was built. PCA was applied to this stack in order to obtain dominant spatial and temporal modes. The truncation only used the first relevant modes that influence measured displacement fields above their standard uncertainty. Then, using a defined number of terms, the procedure updated the constructed stack for correcting the measured displacement fields. The whole procedure required similar computation time as conventional 
DIC analyses.

Five Wedge Splitting Tests (WSTs) were analyzed to measure Notch Opening Displacements (NODs) at room 371 temperature and $600^{\circ} \mathrm{C}$. The cases presented increased challenges (due to speckle pattern extinction, changes in illu- 372 mination, and shadows) that allowed the robustness of the proposed methodology to be probed. The latter was also 373 applied to a dilatometry experiment in which very severe speckle pattern extinction occurred. It was shown that the present method drastically reduced the gray level residuals in DIC analyses. Additionally, this simple proposition to $\mathbf{3 7 5}$ obtain reference states suited for each time step can be implemented non-intrusively in any DIC code (i.e., by only 376 changing the input images for the registrations).

Since global measurements were sought such as NODs for the investigated WSTs and Coefficient of Thermal 378 Expansion (CTE) for the dilatometry experiment, the proposed method did not impact much on their calculation. This 379 fact is believed to be related to the reduced kinematic basis chosen herein to explore how heat haze, illumination, and 380 speckle changes could directly affect the gray level residuals. Last, quasi-direct DIC (i.e., updating the reference image when divergence was detected) proved to be a very robust procedure to deal with such difficult cases in which the gray $\mathbf{3 8 2}$ level residuals increased up to a point that the images could be deemed lost for conventional DIC analyses.

A straightforward perspective is the application of such a procedure in cases that may present variations in the gray $\mathbf{3 8 4}$ levels of the imaged pixels (e.g., from speckle and illumination changes that may be not be experimentally avoided in $\mathbf{3 8 5}$ some setups, to the presence of cracks, oxidation, localized strains, phase transformation phenomena). Depending on 386 the investigated case, the temporal and spatial modulations obtained via PCA may give insight into the phenomenon 387 that is changing the acquired images.

\section{Acknowledgments}

This study was financed in part by the Coordenação de Aperfeiçoamento de Pessoal de Nível Superior - Brasil (CAPES) - Finance Code 001 CAPES (Brazil) and \#2018/15266-0 and \#2018/23081-0, São Paulo Research Foundation (FAPESP). The authors would like to thank IBAR (Indústrias Brasileiras de Artigos Refratários, Poá, Brazil) for kindly providing the materials.

\section{Credit authorship statement}

R. Vargas: Conceptualization, Methodology, Investigation, Software, Original draft preparation, Writing - Review

R.B. Canto: Supervision, Writing - Review \& Editing, Resources, Funding acquisition, Project administration

F. Hild: Supervision, Conceptualization, Methodology, Software, Formal analysis, Writing - Review \& Editing, 
On accounting for speckle extinction for DIC via PCA

S. Roux: Supervision, Conceptualization, Methodology, Formal analysis, Writing - Review \& Editing

\section{Declaration of Competing Interest}

The authors declare that they have no known competing financial interests or personal relationships that could have

\section{References}

[1] M. Sutton, J. Orteu, H. Schreier, Image correlation for shape, motion and deformation measurements: Basic Concepts, Theory and Applications, Springer, New York, NY (USA), 2009.

[2] M. Sutton, Computer vision-based, noncontacting deformation measurements in mechanics: A generational transformation, Applied Mechanics Reviews 65 (AMR-13-1009) (2013) 050802.

[3] M. Sutton, F. Hild, Recent advances and perspectives in digital image correlation, Experimental Mechanics 55 (1) (2015) 1-8.

[4] F. Hild, S. Roux, Digital image correlation, in: P. Rastogi, E. Hack (Eds.), Optical Methods for Solid Mechanics. A Full-Field Approach, Wiley-VCH, Weinheim (Germany), 2012, pp. 183-228.

[5] J. S. Lyons, J. Liu, M. A. Sutton, High-temperature deformation measurements using digital-image correlation, Experimental Mechanics 36 (1) (1996) 64-70.

[6] B. Lucas, T. Kanade, An iterative image registration technique with an application to stereo vision, in: 7th International Joint Conference on Artificial Intelligence, 1981, pp. 674-679.

7] S. L. Brunton, J. N. Kutz, Data-driven science and engineering: Machine learning, dynamical systems, and control, Cambridge University Press, 2019.

8] C. A. Whyte, B. Stojadinovic, R. Pless, Motion component analysis of a squat reinforced concrete shear wall, in: Proceedings of the 9th European Conference on Structural Dynamic (EURODYN 2014), Faculty of Engineering of University of Porto, 2014, pp. $2151-2156$.

[9] S. N. Grama, S. J. Subramanian, Computation of full-field strains using principal component analysis, Experimental Mechanics 54 (6) (2014) 913-933.

[10] W. Hao, J. Zhu, Q. Zhu, L. Chen, L. Li, Displacement field denoising for high-temperature digital image correlation using principal component analysis, Mechanics of Advanced Materials and Structures 24 (10) (2017) 830-839.

[11] N. R. Hugenberg, L. Dong, J. A. Cooper, D. T. Corr, A. A. Oberai, Characterization of spatially graded biomechanical scaffolds, Journal of biomechanical engineering 142 (7) (2020).

12] L. Manzari, P. Göransson, J. Cuenca, I. L. Arteaga, Toward fully anisotropic viscoelastic material models using an automated high-speed optical rig, in: Journal of Physics: Conference Series, Vol. 1149, IOP Publishing, 2018, p. 012003.

[13] C. Jailin, Full field modal measurement with a single standard camera, Optics and Lasers in Engineering 107 (2018) $265-272$.

[14] B. Liu, Y. Zhou, Y.-T. Gao, C. Xu, Experimental and numerical study on crack development characteristics between two cavities in rock-like material under uniaxial compression, Theoretical and Applied Fracture Mechanics 109 (2020) 102755.

15] M. Berny, C. Jailin, A. Bouterf, F. Hild, S. Roux, Mode-enhanced space-time DIC: Applications to ultra high-speed imaging, Measurement Science and Technology 29 (12) (2018) 125008.

16] K. Han, M. Ciccotti, S. Roux, Measuring nanoscale stress intensity factors with an atomic force microscope, EPL (Europhysics Letters) 89 (6) 
[17] J. Neggers, J. P. M. Hoefnagels, F. Hild, S. Roux, M. G. D. Geers, Direct stress-strain measurements from bulged membranes using topography image correlation, Experimental Mechanics 54 (5) (2014) 717-727.

[18] A. Maynadier, M. Poncelet, K. Lavernhe-Taillard, S. Roux, One-shot measurement of thermal and kinematic fields: Infra-Red Image Correlation (IRIC), Experimental Mechanics 52 (3) (2011) 241-255.

[19] A. Charbal, S. Roux, F. Hild, L. Vincent, Regularised digital-level corrections for infrared image correlation, Quantitative InfraRed Thermography Journal 15 (2) (2018) 172-193.

[20] B. Peng, Q. Zhang, W. Zhou, X. Hao, L. Ding, Modified correlation criterion for digital image correlation considering the effect of lighting variations in deformation measurements, Optical Engineering 51 (1) (2012) 017004.

21] G. Gu, Y. Wang, Uneven intensity change correction of speckle images using morphological top-hat transform in digital image correlation, The Imaging Science Journal 63 (8) (2015) 488-494.

2] X. Hu, G. Wu, Z. Xie, F. Liu, Position-based intensity change model for illumination variation in digital image correlation, Optical Engineering 59 (2) (2020) 024102.

23] B. Pan, H. Xie, Z. Wang, Equivalence of digital image correlation criteria for pattern matching, Applied Optics 49 (28) (2010) $5501-5509$.

[24] W. Tong, Formulation of Lucas-Kanade Digital Image Correlation Algorithms for Non-contact Deformation Measurements: A Review, Strain 49 (4) (2013) 313-334.

25] B. Wang, B. Pan, G. Lubineau, Some practical considerations in finite element-based digital image correlation, Optics and Lasers in Engineering 73 (2015) 22-32.

26] J. Zhao, P. Yang, Y. Zhao, Neighborhood binary speckle pattern for deformation measurements insensitive to local illumination variation by digital image correlation, Applied optics 56 (16) (2017) 4708-4719.

27] X. Hu, Z. Xie, F. Liu, Estimating gray intensities for saturated speckle to improve the measurement accuracy of digital image correlation, Optics and Lasers in Engineering 139 (2021) 106510.

28] V. F. Sciuti, R. B. Canto, J. Neggers, F. Hild, On the benefits of correcting brightness and contrast in global digital image correlation: Monitoring cracks during curing and drying of a refractory castable, Optics and Lasers in Engineering 136 (2021) 106316.

[29] T. Archer, P. Beauchene, C. Huchette, F. Hild, Global digital image correlation up to very high temperatures with grey level corrections, Measurement Science and Technology 31 (2) (2019) 024003.

0] G. Hallo, C. Lacombe, J. Néauport, F. Hild, Detection and tracking of laser damage sites on fused silica components by digital image correlation, Optics and Lasers in Engineering 146 (2021) 106674.

11] G. F. Bomarito, J. D. Hochhalter, T. J. Ruggles, Development of optimal multiscale patterns for digital image correlation via local grayscale variation, Experimental Mechanics 58 (7) (2018) 1169-1180.

[32] N. Dematteis, D. Giordan, Comparison of digital image correlation methods and the impact of noise in geoscience applications, Remote

Sensing 13 (2) (2021) 327.
3] R. Vargas, X. Pinelli, B. Smaniotto, F. Hild, R. Canto, Effect of sintering temperature on fracture energy of Alumina-Mullite-Zirconia castable

at $600^{\circ} \mathrm{C}$, Journal of the European Ceramics Society (2021) in press.
34] B. Wagne, S. Roux, F. Hild, Spectral approach to displacement evaluation from image analysis, European Physical Journal AP 17 (2002) $247-252$.

5] G. Besnard, F. Hild, S. Roux, "Finite-Element" displacement fields analysis from digital images: Application to Portevin-Le Chatelier bands, Experimental Mechanics 46 (6) (2006) 789-803.

[36] H. Leclerc, J. Neggers, F. Mathieu, F. Hild, S. Roux, Correli 3.0, IDDN.FR.001.520008.000.S.P.2015.000.31500, Agence pour la Protection 
des Programmes, Paris (France) (2015).

[37] M. Berny, T. Archer, A. Mavel, P. Beauchêne, S. Roux, F. Hild, On the analysis of heat haze effects with spacetime DIC, Opt. Lasers Eng. 40 474 (2018) 544-556.

[38] S. Ribeiro, C. C. D. Exposito, J. A. Rodrigues, Projeto, adaptação, instalação e testes preliminares para um sistema de medida de energia de fratura de materiais cerâmicos pelo método da cunha, Cerâmica 54 (2008) 418-426.

[39] R. Vargas, J. Neggers, R. B. Canto, J. A. Rodrigues, F. Hild, Comparison of two full-field identification methods for the wedge splitting test on a refractory, Journal of the European Ceramic Society 38 (16) (2018) 5569 - 5579.

[40] M. C. Greca, J. V. Emiliano, A. M. Segadães, Revised phase equilibrium relationships in the system $\mathrm{Al}_{2} \mathrm{O}_{3}-\mathrm{ZrO}_{2}-\mathrm{SiO}_{2}$, Journal of the European Ceramic Society 9 (4) (1992) 271-283.

[41] W. D. Callister, Ciência e Engenharia de Materiais: Uma Introdução Ed, 7th Edition, 2007. 